\title{
The genus Stylosanthes (Fabaceae, Papilionoideae, Dalbergieae) in South America
}

\author{
RICARDO O. VANNI ${ }^{1}$
}

\begin{abstract}
Summary: The papilionoid genus Stylosanthes Sw. includes about $50 \mathrm{spp}$. distributed world wide in the tropics, approximately half of them grow in South America.The present study focuses on South American Stylosanthes. Based on examinations of herbarium specimens, as well as field observations, a total of 25 taxa (23 spp. and 2 varieties) are here described and identified with a key. Most of the species have been found to be more widely distributed than expected from the previous taxonomic literature, and the genus appears to be mainly concentrated in Brazil and Paraguay. Stylosanthes leiocarpa Vogel is new to the flora of Argentina and the presence of S. nervosa J. F. Macbr. is confirmed in Argentina. The nomenclature of S. guianensis (Aubl.) Sw. is analyzed. Clarifications are made about nine recently described Brazilian species. The names S. hispida Rich. and S. longiseta Micheli are resurrected. Lectotypes or neotypes for eight species and 18 new synonyms are proposed.
\end{abstract}

Key words: Leguminosae, South America, Stylosanthes, taxonomy, distribution, nomenclature.

Resumen: El género Stylosanthes (Fabaceae, Papilionoideae, Dalbergieae) en Sudamérica. En el género Stylosanthes se incluyen 50 spp., distribuídas entre los trópicos, aproximadamente la mitad de ellas viven en Sudamérica. El presente estudio trata las especies sudamericanas. Basados en el estudio de ejemplares de herbario y observaciones en el campo, se describe un total de 25 taxones (23 spp. y dos variedades), los cuales se identifican con una clave. Se amplía la distribución de la mayoría de las especies, referente a la literatura anterior, determinando centros de variación en Brasil y Paraguay. Stylosanthes leiocarpa Vogel es novedad para la flora Argentina y se confirma S. nervosa J. F. Macbr. para este país. Se analiza la nomenclatura de Stylosanthes guianensis (Aubl.) Sw. Se clarifican las nueve especies recientemente descriptas para Brasil. Se rehabilitan los nombres S. hispida Mich. y S. longiseta Micheli. Se leptotipifican o neotipifican 8 especies y se proponen 18 nuevos sinónimos.

Palabras clave: Leguminosae, Sudamérica, Stylosanthes, taxonomía, distribución, nomenclatura.

\section{INTRODUCTION}

The genus Stylosanthes Sw. is distributed pantropically, with most species occurring in the Americas and few species in Africa, Asia, and Malaysia, while in Australia there are only a number of introduced species. The exact number of species in the genus is actually unclear. According to Lewis et al. (2005) there are probably some 25 species, but up to 50 species exist in the literature. As a matter of fact, the taxonomic history of Stylosanthes is

\footnotetext{
${ }^{1}$ Instituto de Botánica del Nordeste (UNNE-CONICET), Fac. Ciencias Agrarias UNNE, Sargento Cabral 2131, 3400 Corrientes; gvanni@agr.unne.edu.ar
}

characterized by difficulties in interpreting and circumscribing species (Mohlenbrock, 1957; 't Mannetje, 1977; Ferreira \& Sousa Costa, 1977; Maass \& 't Mannetje, 2002; Calles \& Schult-Kraft 2010). Swartz (1788) first described the genus from the Caribbean, Taubert (1890) is the first to provide a comprehensive revision of the genus worldwide, recognizing 22 species, and including identification keys and species descriptions.

In the Americas, the geographical distribution of Stylosanthes ranges from the southern United States to Uruguay, with at least two main centers of diversification, one in Mexico with seven species and the other in central Brazil with eight species.

Mohlenbrock (1957) provided a revision of Stylosanthes mentioning 25 species mainly 
distributed in the Neotropics. For the flora of Argentina, Mohlenbrock (1957) cites three taxa $S$. bracteata Vogel (but with doubts and based only on two specimens collected by Spegazzini), $S$. nervosa J. F. Macbr., and S. guianensis. He considered $S$. gracilis, S. hippocampoides Mohlenbr., and $S$. montevidensis Vogel (as well as its synonym $S$. juncea) as synonyms of $S$. guianensis, but without examining the respective type specimens. Also, he did not study Argentinean specimens of $S$. macrosoma.

The objective of this work, was to study and define the 25 species of Stylosanthes of South America develop key for proper identification, descriptions, illustrations and distribution maps.

\section{Material and Methods}

Herbarium material was examined, including most of the specimens of species cited by Vogel (1838) and Taubert (1890), from collections of the most important herbaria in North America (NY, US), Europe (B, BR, C, G, K, M, P), Brazil (MBM, PAMG, RB, SP), and Argentina (BA, BAB, CTES, CORD, LIL, LP, SI).

Living specimens were observed in the field and plant material was collected and fixed for cytological analyses in $S$. nervosa since its chromosome number was unknown $2 \mathrm{n}=20$ chromosomes. Cytological analyses used for $S$. scabra and S. seabrana B. L. Maass \& 't Mannetje followed the protocol described in Vanni \& Fernandez (2011). Seeds were scarified in hot water at $75^{\circ} \mathrm{C}$ for ten minutes. After germination, seedling roots were treated in 8-oxiquinolein for three hours, fixed in acid lactic absolute-alcohol for 24 hours, and stored in $70 \%$ alcohol. Root tips were hydrolyzed for eight minutes in 1N HCL at $60^{\circ}$ and were stained in Feulgen (Fernandez, 1973). Roots were macerated and subsequently modified in a lacto-acetic acid of orcein. The material was examined during the metaphase stage of cell division using light microscopy.

\section{TAXonomic Treatment}

Stylosanthes Sw., Prodr. (Sw.) 7: 108. 1788. TYPE: Hedysarum hamatum L. = Stylosanthes hamata (L.) Taub.
Stylosanthes procumbens Sw., lectotype designated by N. L. Britton \& A. Brown, Ill. Fl. N.U.S., ed.2 2: 393(1913) nom. illeg. (Hedysarum hamatum L., S. hamata (L.) Taub., Kirkbride et al., 1987).

Perennial, with branches herbaceous to lignified, indument throughout with hairs normally whitish and fine, bristles with multi cellular base, glandular, sometimes secreting a droplet from apex. Leaves trifoliate. Stipules united, persistent. Inflorescence a spike, elongated or small, globose, 1-to30flowered. Flowers with hypanthium at anthesis $5.5 \mathrm{~mm}$ long (at bud stage extremely short); calyx inserted at hypanthium apex, tubular and campanulate, with 5 ciliated teeth, the 2 superior totally united; corolla yellow, with unguiculate petals; androecium surrounding terminal part of style with stigma. Gynophora present at base of hypanthium, ovary bi-ovulate, style as long as hypanthium following curvature of androecial tube, style dilated apically below stigma. Entire structure, especially hypanthium, allows flower to emerge from spike in centripetal direction. Only 1 flower per day and per spike is usually open. The hypanthium and its bearing parts fall after fertilization. Fruit loment with 1 or 2 fertile segments, style persistent, forming a beak, variable in form and size. $2 \mathrm{n}=20,40$.

Distribution, habitat, and phenology. Stylosanthes is found in natural pastures, savanna-like habitats, cerrados, mountain zones, and understory vegetation. Flowering: November through April. Fruiting: March and April.

Obs.I. Swartz created the genus Stylosanthes in 1788, with two species: S. procumbens, and S. viscosa. Stylosanthes procumbes Sw., was a superfluos renaming of Hedysarum hamatum L. Swartz cited the Linnean binomial, and mentioned the Sloane illustration (1707 tab. 119, fig. 2).

Obs. II. Stylosanthes plants are highly appreciated by cattle and are in fact commonly called the subtropical alfalfa. Five species are cultivated and used as forage plants in the subtropics where soils are poor in phosphorus: S. guianensis, S. hamata, S. hippocampoides, S. humilis Kunth, and S. scabra (Jansen \& Edye, 1996). 


\section{R.O. Vanni -The genus Stylosanthes in South America}

Obs. III. Stylosanthes was previously included in tribe Hedysareae (Mohlenbrock, 1957). Recent molecular phylogenetic studies place Stylosanthes within tribe Dalbergieae, suggesting a close relationship with the genera Chapmannia Torr. \& A Gray and Arachis L. (Lewis et al., 2005; Simon et al., 2009), the inflorescences of Stylosanthes are spikes, usually elongated or small spikes. In all species, the flowers have a hypanthium and a biovulate ovary, in which one or both ovules can be fertilized according to the species. The fruit is a non-geocarpic loment. In Arachis, inflorescences are usually small spikes, and flowers have a conspicuously developed hypanthium. The ovary is usually biovulate; the fruit is geocarpic with one or two seeds. Stylosanthes consistently bears three leaflets per leaf, whereas Arachis usually bears four leaflets per leaf, except species of Arachis sect. Trierectoides Krapov. \& W. C. Greg., which consists of erect herbs with three leaflets per leaf. Arachis sect. Trierectoides appears genetically well separated from the rest of the sections in Arachis and represents the most primitive lineage (Krapovickas \& Gregory, 1994). The species in this section are $A$. guaranitica Chodat \& Hassl. and $A$. tuberosa Bong. ex Benth., with an important center of diversification in the Amambay Mountains, Paraguay, coinciding with one of the diversification centers of Stylosanthes.

Obs. IV. The geographic distribution of most species is now extended with respect to the previous literature (Mohlenbrock, 1957). According to Mohlenbrock, these species were especially concentrated in Brazil, with some occurring also in Paraguay, and only four occurring also in Argentina and Uruguay. According to this study, there is an additional possible center of diversification in Paraguay (Fig. 1). S. scabra, which was cited only for Bolivia, Brazil, Colombia, Ecuador and Venezuela (Mohlenbrock, 1957), is shown to be one of the most widely distributed species (Fig. 2). Stylosanthes capitata Vogel, S. hispida Rich., and $S$. longiseta Micheli were previously known only from Venezuela and Brazil (Mohlenbrock, 1957), and their distribution now extends from northern South America to Bolivia and Paraguay. Stylosanthes bracteata is here excluded from the flora of Argentina, but occurs in Brazil and Paraguay and is newly cited for Bolivia.

Obs. $V$. The type and shape of the fruit is important to define the species of Stylosanhes. Fruits of some species are illustrated in Fig. 3.

\section{Key to the Species of Stylosanthes in South America}

1. Fruit with 2 developed segments.

2. Fruit segments glabrous, beak developed, with a slight apical curvature, few small hairs in the concavity or at the base of the beak.

3. Spikes elongated, up to 8-flowered, without ramifications at the base, exceptionally contracted. Plants with few or no bristles.

9. S. leiocarpa

3'. Spikes globose, dense, terminal, 15 or more flowers. Plants with bristles throughout, hispid, erected.

6. S. hispida

$2^{\prime}$. Fruit segments with hairs variable in color and density.

3. Fruit beak apically spiral, both segments or only the inferior with ferrugineous hairs.

4. Leaflets $14-20 \times 3-8 \mathrm{~mm}$, elliptic.

3. S. capitata

$4^{\prime}$. Leaflets 7-12 × 1.5-3 mm, narrowly elliptic.

5. Fruit ca. $4 \mathrm{~mm}$ long.

13. S. maracajuensis

5'. Fruit ca. $11.5 \mathrm{~mm}$ long.

15. S. nervosa 


\section{Bol. Soc. Argent. Bot. 52 (3) 2017}

3'. Fruit beak only slightly curved apically, hairs of other colors.

4. Fruit segments with white, dense hairs.

5. Fruit beak forming a very short open hook.

19. S. scabra

5 '. Fruit beak forming a wider, closed hook.

6a. Plants with basally thickened bristles.

22. S. tuberculata

6b. Plants with whitish hairs, lacking bristles.

20. S. sericeiceps

4'. Fruit segments with yellowish dense hairs.

5. Inferior segment with dense yellowish hairs, superior segment almost glabrous. Beak of $4 \mathrm{~mm}$ long, slightly curved apically.

8. S. humilis

$5^{\prime}$. Both segments with dense hairs. Beak curly, closed, $2 \mathrm{~mm}$ long.

21. S. sympodialis

$1^{\prime}$. Fruit with a single developed segment, sometimes 2 segments in $S$. viscosa.

2. Plants with few leaves; most branches erect.

3. Spikes1-to 18-flowered.

4. Leaves digitate-trifoliate. Fruit segment oblong, cuneate, castaneous when ripe, $3 \times 2 \mathrm{~mm}$. Beak short, $0.2 \mathrm{~mm}$, hook-shaped.

10. S. linearifolia

4'. Leaves pinnate trifoliate.

5. Spikes globose up to 7-flowered. Leaflets narrowly elliptic.

12. S. macrosoma

5'. Spikes elongated, up to 18 -flowered, up to $55 \mathrm{~mm}$ long, terminal, rarely axillary, in some cases 2 spikes per axis. Leaflets linear, 20-30 $\times 0.5-1 \mathrm{~mm}$.

1. S. angustifolia

$3^{\prime}$. Spikes up to 30 -flowered.

14. S montevidensis

2 '. Plants densely leafy, variable in habit; branches suberect to prostrate.

3. Bracts ca. $13 \times 9 \mathrm{~mm}$, membranous, wrinkled, straw-colored, apically tridentate.

2. S. bracteata

$3^{\prime}$. Bracts ca. $4 \times 7 \mathrm{~mm}$.

4. Fruit beak straight, never curved or curly.

16. S. recta

$4^{\prime}$. Fruit beak reduced in a small appendix of $0.5 \mathrm{~mm}$ long.

5. Leaflets narrowly elliptic. Upper side of the fruit with white hairs.

4. S. guianensis

6a. Plants densely leafy, branches decumbent or suberect, hairs incurved and dense, yellowish and bristles. Inflorescences 8-flowered. Central and South America.

4a. S. guianensis var. guianensis 


\section{R.O. Vanni -The genus Stylosanthes in South America}

6b. Plants with few leaves, branches erect, hairs curved variably dense, whitish, bristles dense, ca. $3.5 \mathrm{~mm}$, yellow. Inflorescences 15-flowered. Central and South America.

4b. S. guianensis var. gracilis

6c. Plants densely leafy with bristles only. Inflorescences 2- to 4-flowered. Mexico.

4c. S. guianensis var. dissitiflora

$5^{\prime}$. Leaflets obovate. Fruit covered with capitate hairs.

11. S. longiseta

$4^{\prime \prime}$. Beak developed, curved, or spiral.

5. Fruit segment pubescent with bristles.

6. Prostrate herbs with dense bristles, indument ferrugineous and sticky. Leaflets 12-19 × 3-5 $\mathrm{mm}$.

23. S. viscosa

$6^{\prime}$. Erect subshrubs, up to $1.5 \mathrm{~m}$. Leaflets $15-45 \times 3-10 \mathrm{~mm}$.

18. S. ruellioides

$5^{\prime}$. Fruit segment with thick, succulent glands, lacking bristles. Plants with bristles and white, curved hairs.

6. Beak closed, strongly spiral.

7. S. hippocampoides

$6^{\prime}$. Beak open, hook-shaped.

17. S. rostrata

$6^{\prime \prime}$. Beak slightly incurved.

5. S. hamata

1. Stylosanthes angustifolia Vogel, Linnaea 12: 63. 1838. TYPE: Brazil, merd., inter Campos et Victoria, inter Victoria et Bahia, Sellow leg. (holotype, B, destroyed). Brazil. Cabo Frio, (Rio de Janeiro), Julio 1877, Glaziou 10511(neotype designated here, P!).

Perennial, branches erect with bristles $2 \mathrm{~mm}$, no hairs. Leaves pinnate trifoliate. Stipules adnate at base of petiole, ending in 2 aristae up to $6 \mathrm{~mm}$ with bristles. Leaflets $20-30 \times 0.5-1 \mathrm{~mm}$, linear, acute; superior side glabrous, inferior side with 3 veins highly conspicuous and bristles. Inflorescence as terminal, elongated spikes, up to $55 \mathrm{~mm}$ long, sometimes axillary, rarely 2 spikes in axil, up to 18 -flowered. Bracts united at stipules, highly vellute with hairs white-yellowish and dense, ending in triangular lamina. Flowers $5 \mathrm{~mm}$. Hypanthium 6 $\mathrm{mm}$. Fruit with only 1 segment, $10 \mathrm{~mm}$, the segment $3.5 \mathrm{~mm}$, with white, weak hairs, beak $7 \mathrm{~mm}$, with a spire at apex, hairs white throughout length.
Distribution and habitat. It is found in Surinam, French Guiana, Venezuela, as well as northern and northeastern Brazil, in grassland savannas, at (80) $100-400(600) \mathrm{m}$ a. s. 1.

Additional specimens examined. BRAZIL. Bahia: Bahia, Martius 2351 (M); Estrada Chapadao de Cima a Sao Bento (mun. Paatinga), Hastchbach et al. 67841 (CTES); $15 \mathrm{~km} \mathrm{~W}$ de Joazeiro, Krapovickas et al. 12931 (CTES); Espigao Mestre, ca. $25 \mathrm{~km} \mathrm{~W}$ of Barreiras, Irwin et al. 31349 (NY); Serra do Acuruá, Lagoa Itaparica $10 \mathrm{~km}$ W of Sao Icnácio, Xique Xique, Harley et al. 19116 (NY). Maranhao: Mun. Loreto Ilha de Balsas, region betw. the Balsas and Paraiba river, Eiten et al. 4622 (NY). Pará: In vic. Santarem, June 1850, Spruce s.n. (M, NY); Salinopolis, Prance et al. 14728 (NY). Pará: Hoffmanzent s.n. (BR); Cabo Frio, (Rio de Janeiro), Paraíba: $28 \mathrm{~km} \mathrm{~S}$ de Joao Pessoa, Krapovickas et al. 12876 (CTES). Piauhí: Brasil, tropical, Gardner 2094 (K). FRENCH 

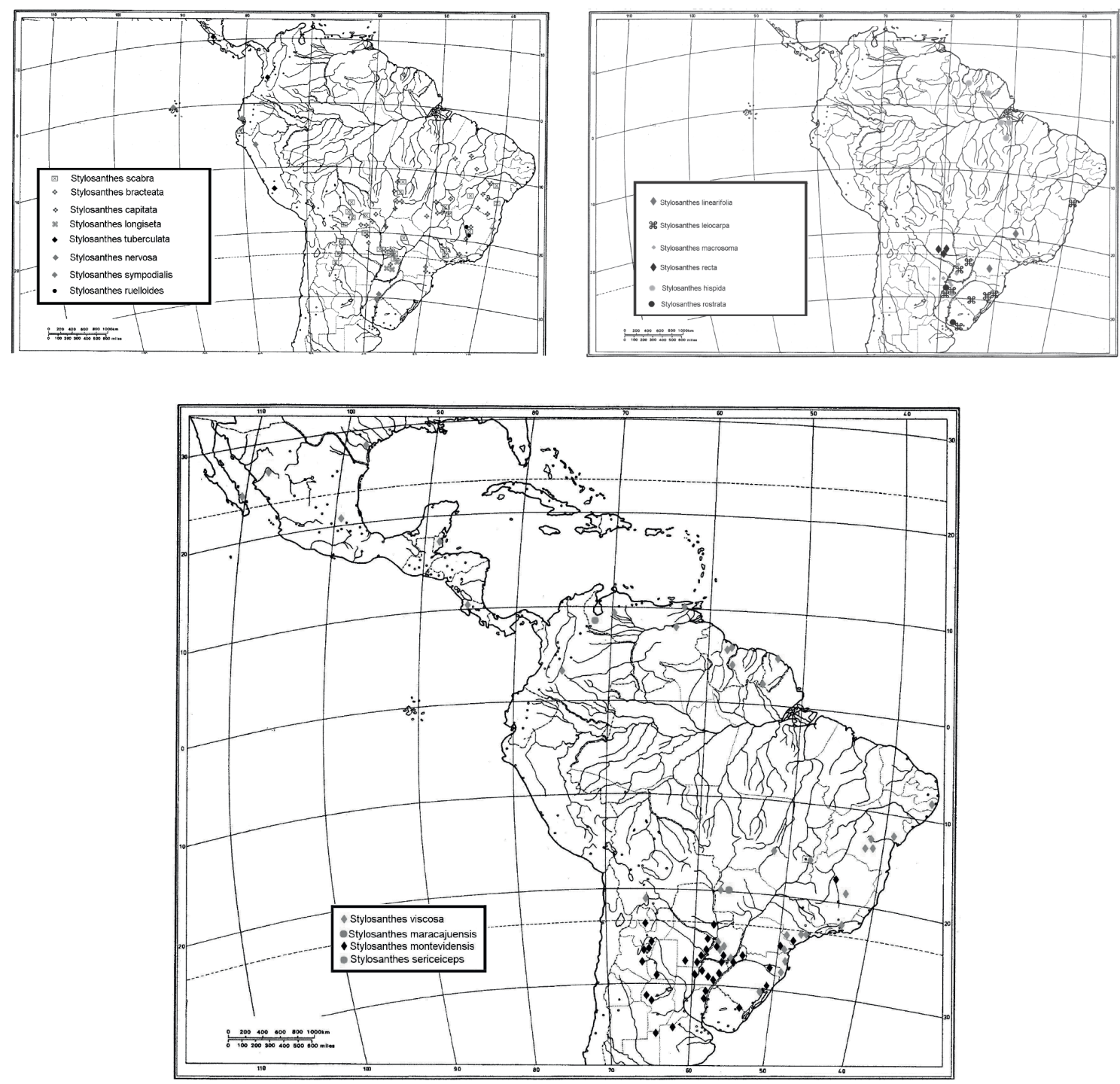

Fig. 1. Distribution maps.

GUIANA. Ferme de bergin, Ansay 46 (BR). GUYANA. British Guyana: Schomburgk 536/816 (K). Rupununi: Distr. Manari, $3^{\circ} 18^{\prime} \mathrm{N}, 59^{\circ} 41^{\prime} \mathrm{W}$, Maas et al. 3659 (GENT); Distr. Manari, JausenJacobs et al. 4402 (B); Karanambo. $3^{\circ} 45^{\prime} \mathrm{N}, 59^{\circ} 20^{\prime} \mathrm{W}$, Maas et al. 7228 (CTES). Rupununi, savanna Lethem, $3^{\circ} 23^{\prime} \mathrm{N}$, 59 $47^{\circ} \mathrm{W}$, Jansen Jacobs 567 (NY); Northern savanna, Goodland 607 (NY); Corossony, 4 km rte. Sinnunary-Iracoubo, Reynal Roquer 21486 (P). SURINAME. Surinam: Wullschlagel 108 (BR). VENEZUELA. Apure: Pedro Cornejo P. N. Santos
Lujardo, río Cinaruco, camino al Orinoco, Castillo et al. 3110 (NY). Bolivar:Dpto. Cedeño, carretera caicada del Orinoco, Pto. Ayacucho, $6^{\circ} 50^{\prime} \mathrm{N}, 66^{\circ} 30^{\prime} \mathrm{W}$, Aymar 3142 (NY)

Obs. Vogel's holotype of this species was not found in any of the herbaria where specimens collected by Sellow are represented. Therefore, the specimen of Glaziou 10511 (P), one of the specimens studied by Taubert (1890) in his monograph, was selected as the neotype for this species. 
R.O. Vanni -The genus Stylosanthes in South America
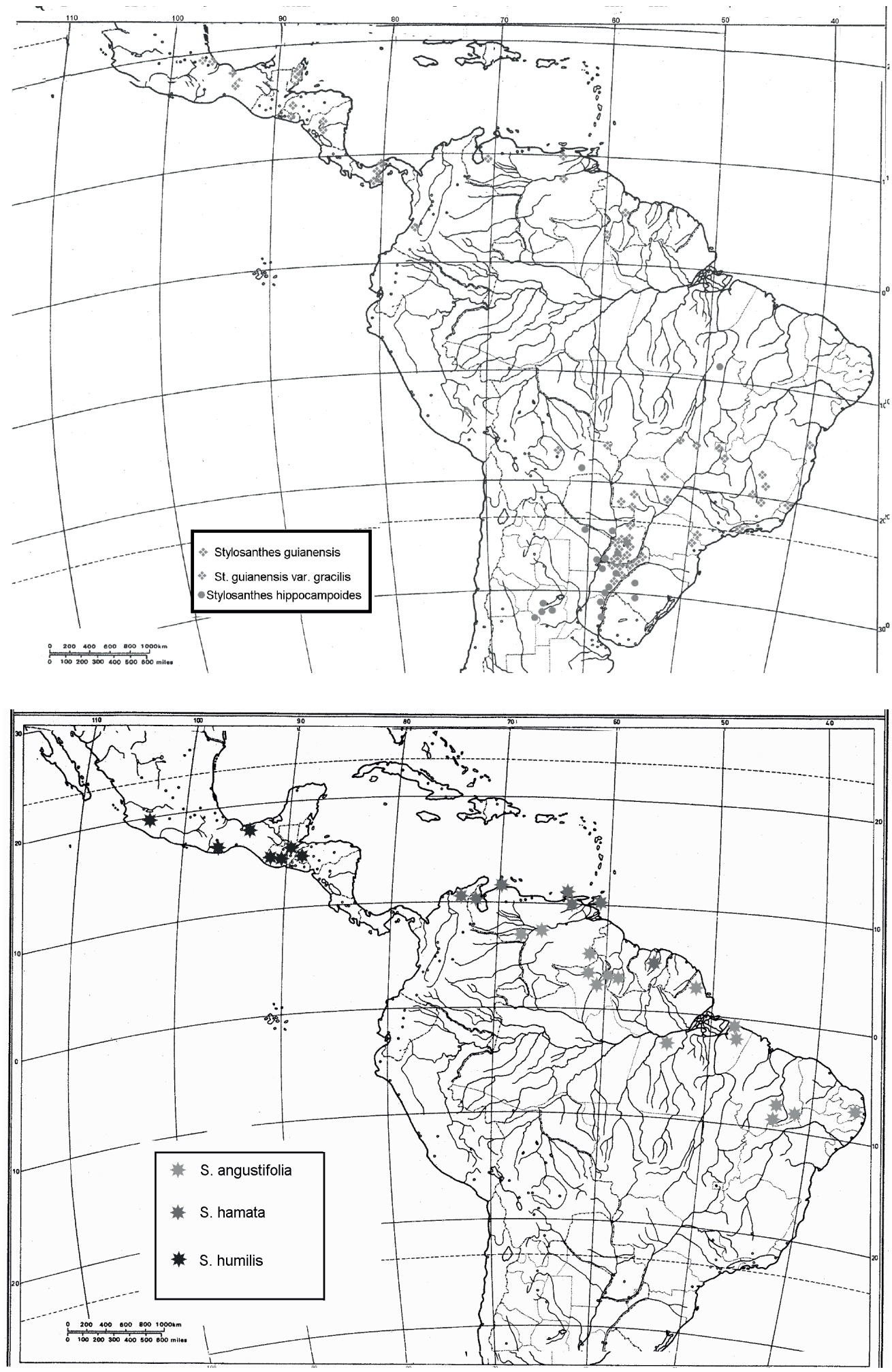

Fig. 2. Distribution maps. 
Bol. Soc. Argent. Bot. 52 (3) 2017

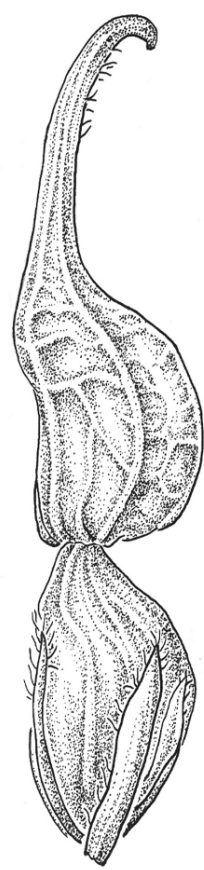

S. leiocarpa

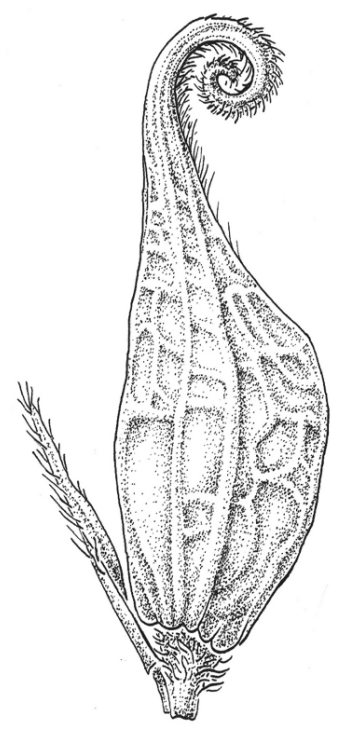

S. macrosoma

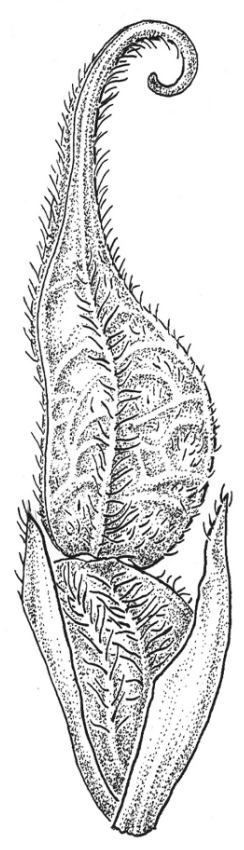

S. nervosa

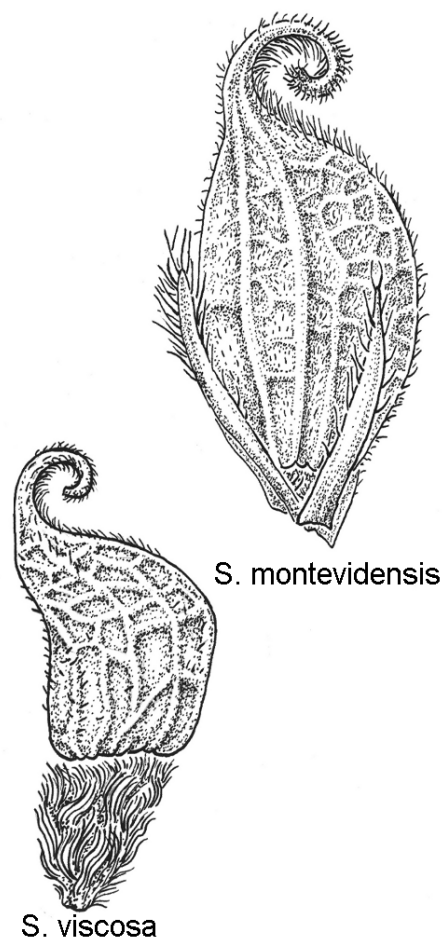

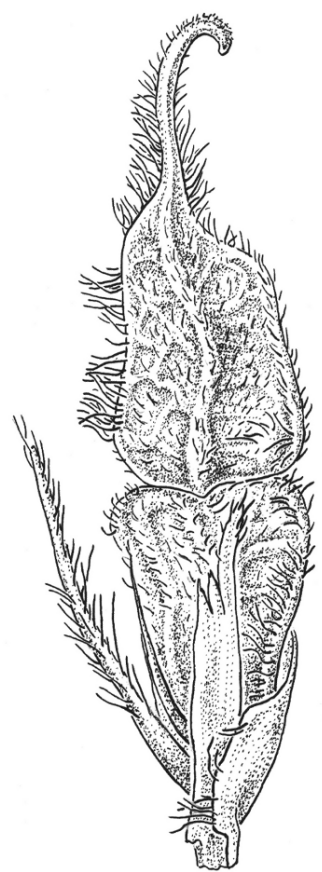

S. scabra

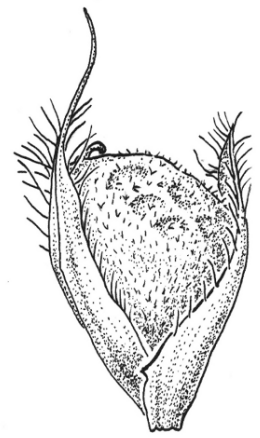

S. guianensis

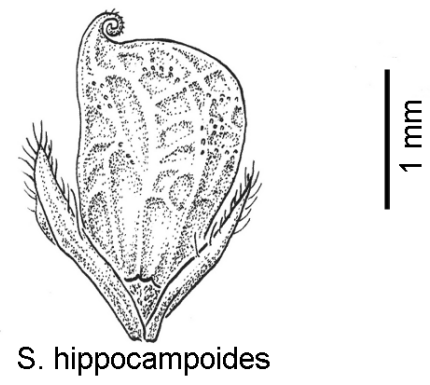

Fig. 3. Fruits some species to Stylosanthes. 
2. Stylosanthes bracteataVogel, Linnaea 12: 70-71. 1838. TYPE: Brazil. Hbt. In Brazil merid. Sellow 4734 (holotype, B, photo 2244 F!).

Perennial, branchs up to $30 \mathrm{~cm}$, some specimens smaller, root system pivotante, woody, highly developed, shoot buds at ground level, hemicryptophyte. Branches with indument with white or weakly tanned, appressed hairs, the hairs abundant throughout the plant. Leaves trifoliate. Stipules $12 \mathrm{~mm}$, adnate at base, with described indument, free at uppermost extremity, where they are point-tipped. Leaflets 11-17 $\times$ 2-3 mm, narrowelliptic, inferior side with highly marked veins, hairs weak, dense, tanned, appressed; indument of superior and inferior side similar. Spikes dense, up to7flowered. Bracts $13 \times 9 \mathrm{~mm}$, membranous, wrinkled, straw-colored, tridentate, hairs ca. $1.5 \mathrm{~mm}$, venation marked. Flowers $6 \mathrm{~mm}$. Hypanthium $10 \mathrm{~mm}$. Calyx $2 \mathrm{~mm}$, campanulate, 5-dentate, the 2 superior teeth united, the inferior tooth largest, all ciliate. Petals unguiculate; standard $6 \mathrm{~mm}$, rounded, claw $2.5 \mathrm{~mm}$ long; wings asymmetric, incurved, lamina rounded, claw $2 \mathrm{~mm}$; keel falcate. Fruit with 1 segment, $8 \mathrm{~mm}$, with hairs ca. $1.5 \mathrm{~mm}$, tanned, dense, beak $3 \mathrm{~mm}$, spiral apically, fruit subtended by a sterile bracteole, pubescent, same length as segment.

Iconography. Burkart, 1939: fig. 13.

Distribution and habitat. It grows in northeastern, central western, and southern Brazil, as well as in Bolivia and Paraguay, in savanna-like habitats.

Additional specimens examined. BOLIVIA. Santa Cruz: Chiquitos, on rute to Roboré, Wood $17400(\mathrm{~K})$; Vogaye D Órbigny dans las Americas Meridionale, Chiquitos, D'Orbigny 871 (P). BRAZIL. Bahia: On rute to Abaica ca. $8 \mathrm{~km} \mathrm{~N}$ of the Town of Rio de Contes, $41^{\circ} 47^{\prime} \mathrm{W}, 13^{\circ} 33^{\prime} \mathrm{W}$, Harley 15218 (NY). Distr. Federal: Confluence Rio Torto, with lagoâ Paranóa, Irwin et al. 9083 (NY); Campus Universitario, perto del Lago, Sucre 761 (NY); Campus Asa Norte Residencial, Anhariguera 35 (NY); Brasilia, Burchel 5680 (K). Brasilia, Sellow s.n. (mentioned in K isotype). Goiás: Serra do Cipó, $17^{\circ} 12^{\prime} \mathrm{S}, 51^{\circ} 47^{\prime} \mathrm{W}, H$. S. Irwin et al. 7576 (NY); Goias, Glaziou 20920 (K); Minas Gerais, in campis graminosis, Riedel 729 (K).
Mato Grosso: Mato Grosso, Gaudichau 1833(P). Mato Grosso do Sul: rio Pardo, in campis siccis arenosis Riedel 440 (K); rio Pardo, Riedel 567 (K, P); Amambai, rd. p/ Cel. Sapucaia, Hatschbach 48411 (C, CTES). Minas Gerais: Minas, Glaziou 16754 (C, K); Lagoa Santa, Warming 3175 (C, P). Paraná: Vila Velha, Krapovickaset al. 40835 (CTES); Mun. Sengés, rio dos Bugres, Hatschbach 35417 (M, Herb. de Pedersen, CTES); Bank of the rio Reis, $5 \mathrm{~km} \mathrm{~S}$ of Jaquariaria, Lindeman et al. 3040 (NY); Ponta Grossa, campo Villa Velha, Hoehne23368 (NY); idem, Hatchsbach 10241 (B); Mun. Ponta Grossa, Buraco do Padre, C. B. Poliguesi et al. 652 (C). São Paulo: São Paulo, Riedel 2047 (K); São Paulo, Sant Hilaire C2 1490 (P). PARAGUAY. Amambay: Cabesera Estrella, Sierra de Amambay, Rojas 1933 (SI); Concepción: Ayo. Primero, in regione cursus superioris fluminis Apa, Hassler 8510 (G); Zwischen rio Apa und Aquidabán, Villa Sana, Fiebrig 5028 (G, K, M, SI); In regione cursus superioris fluminis Apa, Hassler 7921 (G). Caaguazú: Caaguazú, cercanías de Yhú, Fernández Cazas, y Molero F.C. 6-406 (G); Estancia Primera, en campo quemado, Joergensen 4877 (C, SI); In campo Apepú, Tapiraguay Hassler 4363 (G, NY).

3. Stylosanthes capitata Vogel, Linnaea 12: 70. 1838. TYPE: Brazil. Hab. in Brazil; inter Victoria et Bahia Sellow leg. (holotype, B, destroyed). Bahiensis, caxoeira et Feira da Conceicao, February 1819, Martius 2209 (neotype designated here, M!).

Stylosanthes macrocephala M. B. Ferreira \& Sousa Costa, Anais Soc. Bot. Brasil 28: 87. 1977 [1978]. TYPE: Minas Gerais: Brasilia de Minas, $2 \mathrm{~km}$ apos a cidades en direcäo a Saô Francisco, 900 m.s.m., 3 May 1975, M. L. Gavilanes 229 (holotype, RB!). syn. nov.

Stylosanthes bahiensis 't Mannetje \& G. P. Lewis. Kew Bull. 37 (1): 125-127. 1982. TYPE:

Brazil. Bahia: Serra Du Acuruá, 5-6 km S de São Ignacio on the rd. to Gentio do Ouro. Disturbed caatinga, 27 Feb.1977, R. M. Harley, S. J-Mayo. R. M. Storr, T. S. Santos \& R. S. Pinehiro 19134 (holotype CEPLAC, isotype, K!). syn. nov.

Perennial, branchs $70 \mathrm{~cm}$, with indument of whitish, incurved hairs and bristles variable in density. Leaves trifoliate, pinnate-trifoliate. Stipules 10-14 mm, adnate welded to petiole, free 
at opposite margin, point tippedapically, with long, lax, and hyaline hairs, and bristles. Leaflets 14-20 $\times 3-8 \mathrm{~mm}$, elliptic, inferior side with white, marked veins, protruding, hairs hyaline, lax and dense, bristles on veins. Superior side with similar hairs to inferior side, bristles absent. Inflorescences in dense spikes, axillary or terminal, over 20 -flowered. Bracts $9 \times 7 \mathrm{~mm}$, very wide, formed by 2 ovoid, acute and papiraceous parts, short lamina between, with dense, yellow-golden hairs, $1 \mathrm{~mm}$. Flowers $6 \mathrm{~mm}$, hypanthium $7 \mathrm{~mm}$; calyx $2 \mathrm{~mm}$, with appressed hairs, 5 teeth, inferior larger. Corolla 6 $\mathrm{mm}$. Petals unguiculate, standard rounded, wings oblong, keel incurved. Fruit $8 \mathrm{~mm}, 2$-articulated, inferior segment $3 \mathrm{~mm}$, with dense, yellow-golden hairs, superior segment $5 \mathrm{~mm}$, glabrous. Beak slightly curved.

Distribution and habitat. It inhabits in French Guyana, Venezuela, Bolivia, Paraguay, and Brazil in savanna-like habitats and forest openings at 300$1100 \mathrm{~m}$ a. s. 1.

Additional specimens examined. BOLIVIA. Santa Cruz: Chiquitos, ca. $80 \mathrm{~km} \mathrm{~S}$. of José de Chiquitos, on rd. Santa Cruz, Wood et al. 14066 (K); Chiquitos, $2 \mathrm{~km}$ E de Roboré, cerca del río Urasibique, $59^{\circ} 45^{\prime} \mathrm{W}, 18^{\circ} 20^{\prime} \mathrm{S}$, Krapovickas et al. 36778 (CTES); Lourdes, $50 \mathrm{~km}$ al S de Roboré, Fuentes et al. 2095 (CTES); Cordillera, Parque Nacional Kaa-Iya, del Gran Chaco, 19 $14^{\prime} 41^{\prime \prime}$, 60²4'33"W, Fuentes et al. 2304 (CTES); Cerro San Miguel, $19^{\circ} 18^{\prime} \mathrm{S}, 60^{\circ} 39^{\prime} \mathrm{W}, 700--833$ m.s.m., Ramella et al. LR 2521 (CTES). BRAZIL. Bahia: in campis, Martius s.n. (M): Bahiensis caxoeira et Feira da Conceicao, Martius 2209 (M): 14 km $\mathrm{N}$ de Barreiras, BR $135,12^{\circ} 12^{\prime} \mathrm{S}, 44^{\circ} 56^{\prime} \mathrm{W}$, Valls et al. 7042 (CTES); Mun. Victoria da Conquista, Veredinha, Hatschbach 47359 (CTES); Mun. de Oliveira dos Brejinhos, rd. Oliveira dos Bejinhos a Macaúbas, BR 122, km 8, Hatschbach et al. 65096 (CTES); Mun. de Urundí, rd. BR-122, próximo a divisa con Ouro Branco, Hatschbach et al. 56529 (CTES); Mun. de Agua Fría, estrada para Cia. de Celulose da Bahia, Messias Santos et al. 178 (CTES); $9 \mathrm{~km}$ de Serrinha, Duarte et al. 390 (NY); Perto de Serrinha, Castellanos 25117 (NY); Chapada occidental do Bahia, 12 $\mathrm{km}$ fromCorrentina, $44^{\circ} 40^{\prime} \mathrm{W}, 13^{\circ} 15^{\prime} \mathrm{S}$, Harley $21877(\mathrm{NY}) ; 7 \mathrm{~km} \mathrm{~S}$ de río das Contrasestrada terra Livramento do Brumado, $41^{\circ} 50^{\prime} \mathrm{W}, 13^{\circ} 38^{\prime} \mathrm{S}$, Lewis et al. 1993 (NY); $5 \mathrm{~km} \mathrm{~S}$ de Cocos, W. Anderson et al. 37085 (NY); Mun. Rio de Contas, Hatschbach 56796 (CTES); Serra do Sincorá W of Barra da Estiva. $41^{\circ} 25^{\prime} \mathrm{W}, 13^{\circ} 40^{\prime} \mathrm{S}$. Harley et al. 20822 (K). Distr. Federal: $1.5 \mathrm{~km} \mathrm{~W} \mathrm{da} \mathrm{escola}$ Fazendaria $15^{\circ} 49^{\prime} \mathrm{N}$, Kirkbride 1623 (CTES); Cerrado, Ribeirao Sobradinho, Irwin et al. 12110 (NY); Brasilia, Catetinho, Santos et al. 1652 (NY). Goiás: Mun. de Formosa, 17 km de JK, BR-020, acceso destilería Brasil Central $15^{\circ} 4^{\prime} \mathrm{S}, 47^{\circ} 5^{\prime} \mathrm{W}$, Krapovickas et al. 38686 (CTES); Mun. Teresina de Goiás, rd. GO-118, próximo a o rio Sucuri, Hatschbach et al. 60313 (CTES); $1 \mathrm{~km} \mathrm{~S}$ da fonte da agua Mineral Idaiá, $15^{\circ} 24^{\prime} \mathrm{S}, 47^{\circ} 28^{\prime} \mathrm{W}$, Kirlbride 1635 (CTES); 26-31 km S of Goiänia, along hwy. BR-153, Davidse et al. 12281 (NY). Maranaö: Ilha dos Botes, $10 \mathrm{~km}$ abaxio Carolina rio Tocantis, Pires et al. 1994 (NY). Mun. De Loreto, Ilha das Balsas, betw. rios Balsa y Parnaiba, ca. $40 \mathrm{~km} \mathrm{~S}$ de Loreto, Eiten et al. 3867 (NY). Mato Grosso: BR-070, 35 $\mathrm{km}$ W de Paredao Grande, Valls et al. 9036 (CTES). Mato Grosso do Sul: Mun. Säo José do Rio Claro, $6 \mathrm{~km}$ da cidade, $56^{\circ} 45^{\prime} \mathrm{W}, 13^{\circ} 33^{\prime} \mathrm{S}$, Rd. S. José do Rio Claro sentido Campos Novos dos Parecís, Pietrobon Silva et al. 3618 (CTES); Corumbá, Mun. Jacadigo, $29.5 \mathrm{~km}$ Sul do posto Eschas estrada do Tamarineiro, Pott et al. 7785 (CTES); Mun. de Corumbá, Serra Urucú, Hatschbach 29529 (CTES).

Minas Gerais: Sierra do Espinhaco, subida via Montividiu, Mun. Monte Azul, Hatschbach et al. 64982(CTES); $25 \mathrm{~km}$ rd. NE Diamantina, $1.5 \mathrm{~km}$ from rio Jequité, Anderson $8722(\mathrm{NY}) ; 3.5 \mathrm{~km}$ by rd. SW of rio Jequetí and Mandanha, Anderson 8928 (NY); Ca. $15 \mathrm{~km}$ E of Diamantina, Irwin et al. 27830 (NY); Belo Horizonte, en las afueras de la ciudad, L. Williams et al. 5834 (SI). Piauí: Mun. de Cristino Castro, caatinga, rd. Bon JesusCastro do Buruti, km 103, 21 June 1983, Coradín s.n. (NY); Piauhi, Carreiras, Lutzelburg 112 (M). FRENCH GUAIANA. Ferme de Combi, pres de Iracombo. Ausay 31 (BR). PARAGUAY. Alto Paraguay: Madrejón, P. N. Defensores del Chaco, $19^{\circ} 21^{\prime} 46.8^{\prime \prime} \mathrm{S}, 60^{\circ} 20^{\prime} 0.68^{\prime \prime} \mathrm{W}$, Mereles 6697 (CTES). Chaco: Cerro León, Cap. Pablo Lagerenza $20^{\circ} 20^{\prime} \mathrm{S}$, $60^{\circ} 20^{\prime} \mathrm{W}$, Ramella L R 2740 (CTES). SURINAM. Saramacca: Exp. Coebiti, Everaarts 1207 (NY). VENEZUELA. Ciudad Bolivar, vic. of the Orinoco, Bailey et al. 1434 (NY). Managas: Punta de Mata, $20 \mathrm{~km}$ W of Jusepin, Mac Kee 10785(P). 
4. Stylosanthes guianensis (Aubl.) Sw., Kongl. Vetensk. Acad. Nya Handl. 10: 301-302. 1789. Basionym: Trifolium guianense Aubl., Hist. Pl. Guiane 2: 776-778; t. 309. 1775.

Astyposanthes guianensis (Sw.) Herter, Revista Sudamer. Bot. 7:209. 1943. TYPE: French Guyana: Sin loc. J. Aublet, s $n$ (holotype P, NY Neg.97!).

Perennial, branches decumbent or erect. Leaves trifoliolate. Stipules partly united at the petiole base. Leaflets 10-27 x 2-6 mm, narrow elliptic. Inflorescences terminal, spikes globose, with basal ramitications, 2-15 flowered. Flowers 6-7 mm. Hypanthium 3-5 mm. Calyx $3 \mathrm{~mm}$, campanulate. Petal unguiculate; standar rounded emarginated. Fruit with only one fertile segment, $4 \mathrm{~mm}$, oblong, some thick hairs on the upper half; beak short, curved, $0,5 \mathrm{~mm}$

\section{4a. Stylosanthes guianensis (Aubl.) Sw.var. guianensis}

Trifolium guianense Aubl., Hist. pl. Guiane 1: 776. 1775. TYPE: Guyana. Aublet, s. $n$. (holotype P, NY Neg. 97!). Figs. 3, 4.

Stylosanthes viscosa Sw. var. acutifolia Benth., Fl. Bras. 15 (1A): 91. 1859. TYPE: Brazil. Bahia: in sabulosis maritimus, Salzmann (holotype K! = Neg. NY 2303!).

Stylosanthes guianensis (Aubl.) Sw. var. subviscosa Benth. ex Martius, Fl. Bras. 15 (1A): 92. 1859. TYPE: Brazil. Bahia, Minas Gerais, ad Corrego do Jaraguá et rio Jequitinhonha, 1836, J. E. Pohl s.n. (holotype K!).

Stylosanthes guianensis (Aubl.) Sw. var. subviscosa (Benth.) Hassler, Repert. Spec. Nov. Regni Veg. 16: 221 1919. TYPE: Paraguay. In campo, pr. fluminis. Corrientes, Dec., E. Hassler 5858 (lectotype designated here, G!). syn. nov.

Stylosanthes guianensis (Aubl.) Sw. var. subviscosa f. viscosisima Hassler, Repert Spec. Nov. Regni Veg.16: 221. 1919. TYPE: Paraguay. In colle, Tobaty, March. E. Hassler 4010 (holotype, G!).

Stylosanthes guianensis (Aubl.) Sw. var. genuina f. esetosa Hassler, Repert Spec. Nov.Regni Veg. 16: 221. 1919. TYPE: Paraguay. Gran Chaco, Santa Elisa, $23^{\circ} 10^{\prime}$ S, Apr. 1903. E. Hassler 2834 (holotype, G!).

Stylosanthes guianensis (Aubl.) Sw. var. vulgaris M. B. Ferreira \& Sousa Costa, Empresa de Pesquisa Agropecuaria de Minas Gerais 45. 1979. TYPE:
Brazil. Minas Gerais: Rodovía Fernando Diaz, 2 kmapós Betím-Sao Paulo, 5 Mar.1976, M. B. Ferreira \& Sousa Costa 310 (holotype, RB!). syn. nov.

Stylosanthes guianensis (Aubl.) Sw. var. microcephala M. B. Ferreira \& Sousa Costa, Empresa de Pesquisa Agropecuaria de Minas Gerais 46. 1979. TYPE: Brazil. Minas Gerais: Caeté, 4 Feb. 1976, M. B. Ferreira \& Sousa Costa 306 (holotype, RB!). syn. nov.

Stylosanthes nunoi Brandão, Daphne 2 (1): 5. 1991. TYPE: Brazil. Mato Grosso: $12^{\circ} 54^{\prime} \mathrm{S}$ $051^{\circ} 52^{\prime} \mathrm{W}, 270 \mathrm{~km} \mathrm{~N}$ of Xavantina, Nov. 1967, J. Ramos \& R.Sousa 66 (holotype RB, isotype, P!). syn. nov.

Perennial, branches decumbent or suberect, with dense, incurved, yellowish hairs and bristles throughout the plant. Leaves trifoliate. Stipules partly united at petiole base, apical part linear, mucronate, ending in 1 bristle. Leaflets 10-15 $\times$ 2-3 mm, narrow-elliptic, terminal leaflet larger; superior side with dense, curved, whitish hairs and bristles; inferior side with indument of superior side, bristles dense and appressed; venation prominent, lighter green than rest of lamina. Inflorescences terminal, spikes short, globose, simple with basal ramifications, 8-flowered. Flowers $6 \mathrm{~mm}$, each flower protected by bracts similar to stipules and 2 hyaline, bracteoles, ciliate at base. Hypanthium 5 $\mathrm{mm}$. Calyx $3 \mathrm{~mm}$, campanulate, inferior tooth acute and largest, others rounded. Petals unguiculate; standard rounded, emarginated. Wings oblong. Keel incurved. Fruit with only 1 fertile segment, 4 $\times 2-2.5 \mathrm{~mm}$, oblong, with white hairs on apical part, some times up to half the segment, beak very small, $0.5 \mathrm{~mm}$, curved.

Distribution and habitat. This variety is found in Central and South America, from Mexico to Argentina in savanna-like habitats and campos cerrados at (100) 260-1350 (1960) m a.s.l. It is cultivated in Colombia, Brazil, Paraguay, Argentina and in Hainan (China).

Additional specimens examined. ARGENTINA. Corrientes: Dpto. Ituzaingó, cercanías al pueblo, Ibarrola 4352 (LIL); 30 km E de Ituzaingó, sobre rte. 12, Krapovickas 7903 (CTES); E El Plata, Meyer 6736 (LIL); Dpto. Berón de Astrada, ayo. 
Bol. Soc. Argent. Bot. 52 (3) 2017

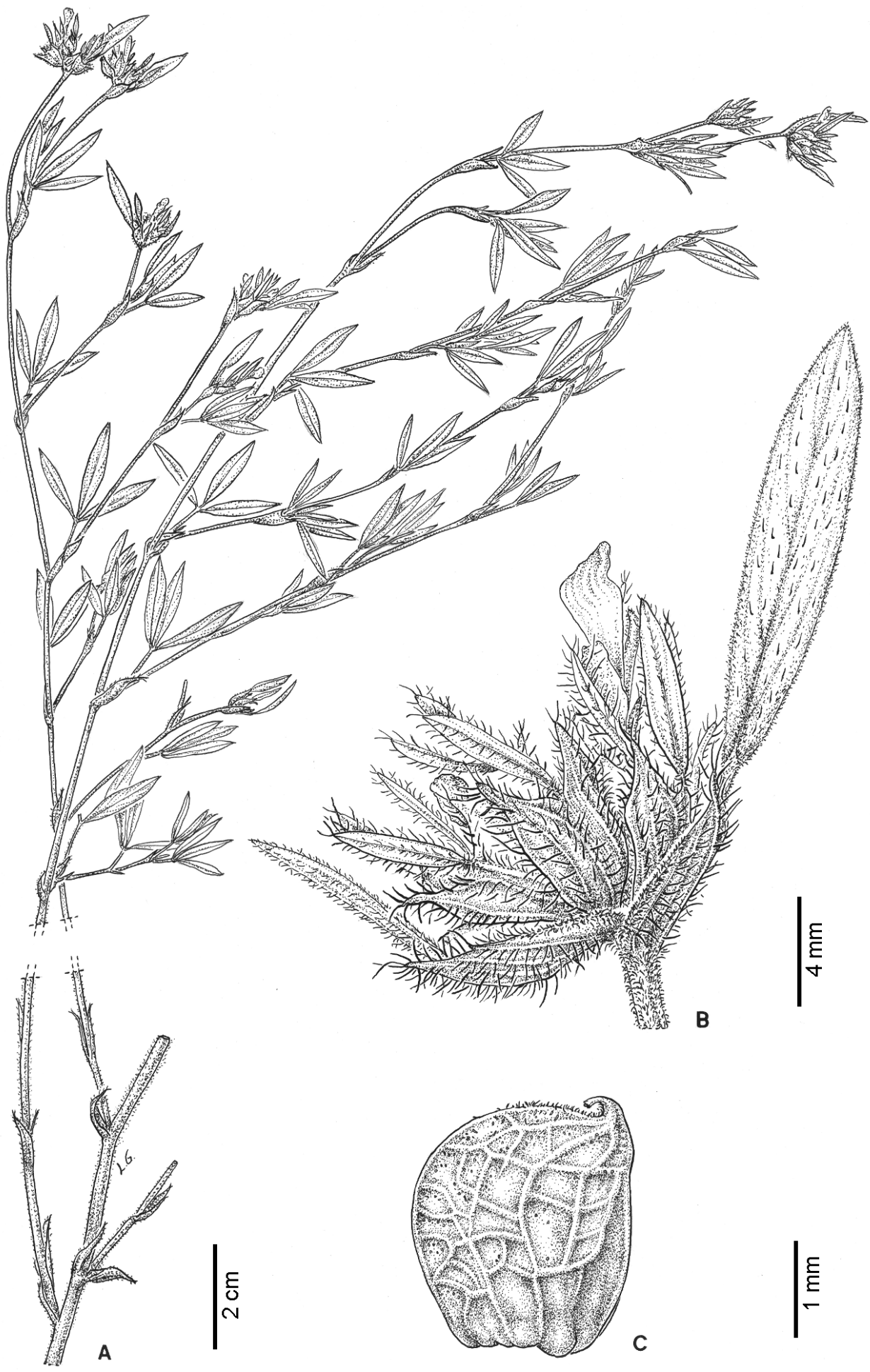

Fig. 4. Stylosanthes guianensis. A. Plant. B. Inflorescence. C. Fruit. (Krapovickas 34504). 
Santa Isabel, sobre ruta 12, Legname et al. 8102 (LIL); Dpto. Santo Tomé, Santo Tomé al N de E Dos Leguas, Ibarrola 1514 (LIL). Dpto. San Martín, Torrent al S, Ibarrola 1682 (LIL). Misiones: Dpto. San Ignacio, casa de Quiroga, Krapovickas et al. 28720 (CTES); Camino a cerro Victoria, Pfeiffer 71 (CTES). BELICE. Maskall: Gentle 1050 (NY); Toledo, Swasey Branch, Monkey River, Gentle 3898 (NY); Starm creek. Melinda, Gentle 1883 (NY). BOLIVIA. Santa Cruz: Prov. Santiesteban, Saavedra, 15 km NNE Montero, Saldías 714 (NY); Guania Tipuani, Bang 1459 (C); Milluguaya, in North Yungas, Buchtien 4105 (C). BRAZIL. Bahia: Mun. Salvador, Itapoa, Batista et al. 796 (NY). Sta. Rosa, Sep.1839, Gadner 2826 (sintype, K!). Distr. Federal: Rio Sao Bartolomeu, immediately E of Paranoá, Irwin et al. 26713 (NY); Brasilia, Martius 855 (BR); Brasilia, Escola Fazendaria, Fiedler 23 (C); Lagoa Santa, Warming 3184-2(C). Mato Grosso do Sul: Alrededores de Campo Grande, camino a Sidrolandia, Krapovickas et al. 34504 (CTES). Minas Gerais: Caldas, Peon da Vista, Martius 384 (BR); Caldas, A. F. Regnell 428(C). Parana: Mun. Pto. Amazonas, Fda. S. Roque, Kummrow 1039 (Herb. de Pedersen, CTES); Rio Jaguariaíva (Mun. Juguariaíva) Praia, BR, Ribas et al. 1739 (C). Sao Paulo: Sebastianopolis, R. J. Z. Martius s. n. (M). COLOMBIA. Cesar: Munred. Serranía La Macarena, Hacienda Los Micos, Mun. San Martín, Bermudez 259 (B). Valle del Cauca: Cali, Universidad del Valle, Silverstone et al. 2436 (NY). COSTA RICA. Concavas: sin loc. C.HLankaster 307 (K); Planta Costaricenses. Pittier 4986 (BR), Pittier 3936 (BR). EL SALVADOR. San Salvador: San Salvador, Calderón 1287 (NY). Vic. of San Salvador, Standley 19398 (NY). FRENCH GUIANA. Cayenne: Savanna Mamaribo, Hoff et al. $5621(\mathrm{P}) ; \mathrm{km} 4$ tornate Cayenne rd., Leuwenberg 11042 (GENT). HONDURAS. Colón: Capuchín, Saunders 816 (NY). Copán: 5 km SW Santa Rosa de Copán, Molina 11707 (NY). El Paraíso: Colinas arenosas de Guayobillas, Molina 13604 (NY). MEXICO. Chiapas: Cerro Brujo, $20 \mathrm{~km}$ S of Ocozocoautla, Bree O. Rove 29102 (NY); Chiapas, $8 \mathrm{~km} \mathrm{~N}$ Del pueblo de Palenque, Mun. De Palenque, Sousa et al. $1200 T(\mathrm{~K})$. Oaxaca: 7 $\mathrm{km}$ SW de Acatepec, carr. Huajuacan-Tehuacan, limite de Puebla, Sousa et al. 7740 (K). Puebla: Orizaba, 1855, Müller s.n. (NY); Montecristo, Matuda 1958 (NY); San Anselma, Gaumer 1955
(C). Vera Cruz: Minatitlán, 15-X-1928, Mell s.n. (NY). NICARAGUA. Chontales: $2 \mathrm{~km} \mathrm{~S}$ of Acoyapa, Sayrnocer 1767 (NY). Matagalpa: Sta. María de Ostuma, cordillera central de Nicaragua, betw. Matagalpa and Jinotega, L. Williams et al. 23486 (NY); $15 \mathrm{~km}$ NE of Matagalpa, along rio Las Cañas, L. Williams et al. 27525 (NY). PANAMA. Herrera: Roadside ca. $3 \mathrm{~km} \mathrm{~N}$ of Las Minas, D'Arcy et al. 14285 (NY). Veracrúz: $270 \mathrm{~km}$ west of Panama city, betw. Santiago and San Felix, Folson et al. 6910 (NY). PARAGUAY. Amambay: Bella Vista, Vanni et al. 286 (CTES); Bella Vista, dry deciduous forest along Rio Apa, Simonis et al. 183 (CTES, U). Caaguazú: 15 km N de Caaguazú, camino a Ihú, Schinini et al. 21960 (CTES). Cordillera: Cerro Tobatí, Degen et al. 555 (CTES). Guairá: Colonia Independencia, $25^{\circ} 45^{\prime} \mathrm{S}, 56^{\circ} 13^{\prime} \mathrm{W}$, Schinini et al. 25172 (CTES). Misiones: $10 \mathrm{~km}$ $\mathrm{N}$ de Ayolas, Schinini et al. 25996 (CTES); Inter rupes, pr. Valenzuela, Hassler 7030 (G); In campis arenosis, cursus sup. fl. Apa, Hassler 8082(G). Pr. Tobaty, Hassler 6454 (G). Inter rupis pr. Piribebuy, Hassler 6882 (G). PERU. Ruiz y Pavon s.n. (B). VENEZUELA. Bolivar: Anzóategui, $\mathrm{S}$ of El Zamuro, $9 \mathrm{~km} \mathrm{NE}$ of Bergantín, $64^{\circ} 17^{\prime} \mathrm{W}, 10^{\circ} 2^{\prime} \mathrm{N}$, Davidse et al. 19356 (NY). Merida: State of Barinas, 2 km from Berinta, Breteler 4195 (GENT).

Obs. I. Stylosanthes guianensis is the most widely distributed species occurring in two of the diversification centers of the genus, Mexico and northeastern Brazil. In this species, taxonomic problems have incurred with species names that different authors have synonymized as $S$. guianensis. Because of the lack of morphological characters that distinguish $S$. guianensis var. vulgaris and $S$. guianensisvar. microcephala from S. guianensis var. guianensis, I consider them synonyms of $S$. guianensis var. guianensis.

Obs. II. Santos et al. (2012), identified S. guianensis varieties using molecular genetic analysis. Their data indicate a high genetic diversity among and within the varieties they studied.

4b. Stylosanthes guianensis (Aubl.) Sw. var. gracilis (Kunth) Vogel, Linnaea 12: 66. 1838. TYPE: Brazil. Brasilia meridionales, VittoriaBahia, Sellow leg. (holotype, K!), ex Herb. Kunth (isotype, B!). Fig. 5. 
Bol. Soc. Argent. Bot. 52 (3) 2017

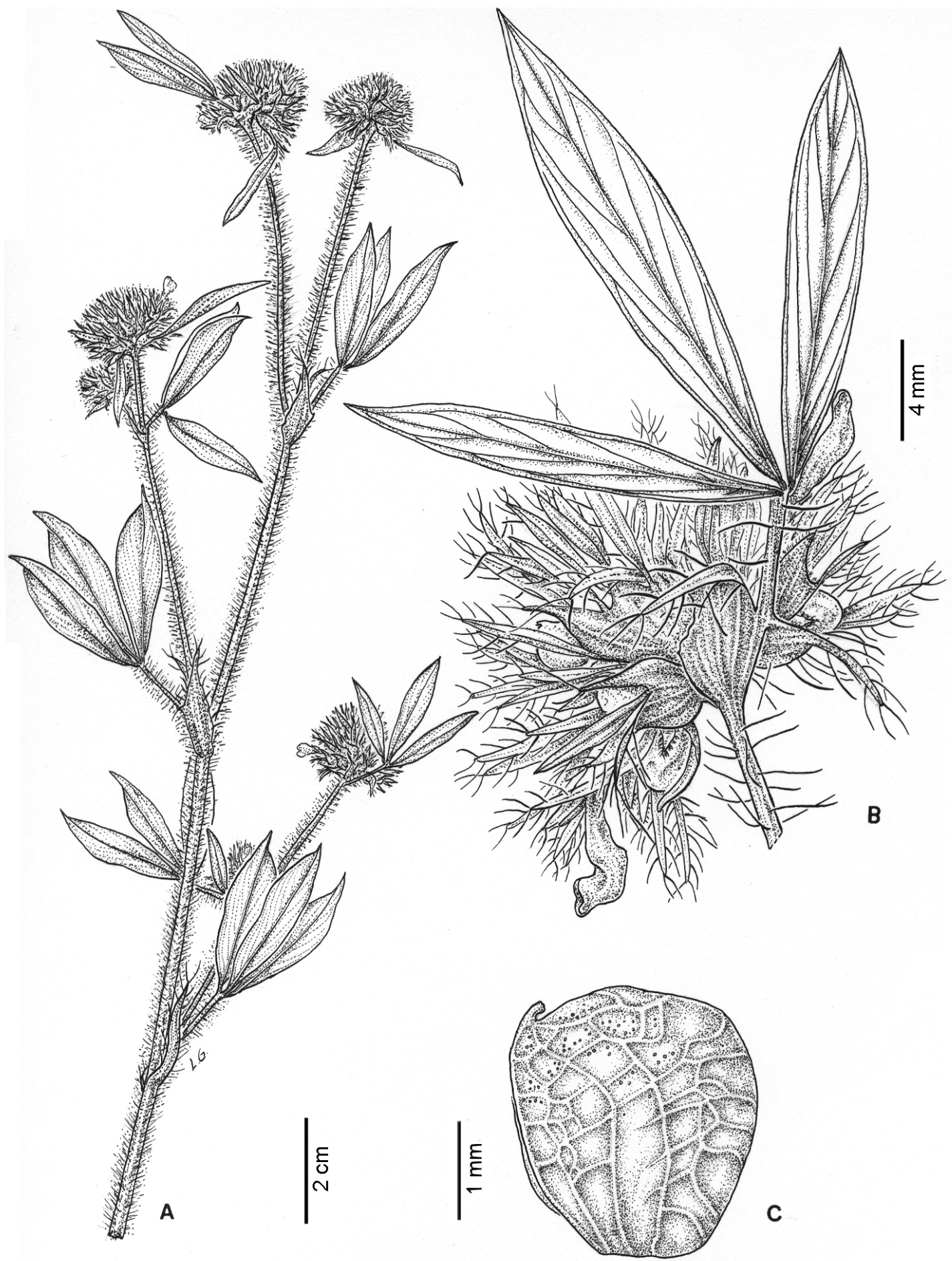

Fig. 5. Stylosanthes guianensis. var. gracilis. A. Plant. B. Inflorescence. C. Fruit. (Hatschbach 34198). 
Stylosanthes gracilis Kunth, Nov. Gen. Sp. 6: 507-508, tab. 596. 1823. TYPE: Venezuela. Caripe, 1799, Humb. \& Bonpl. 314 (holotype, P!).

Stylosanthes guianensis (Aubl.) Sw. var. pubescens Pilger, Bot. Jahrb. Syst. 30: 160. 1901. TYPE: Brazil. Mato Grosso, 1899, Pilger 625 (holotype, B!).

Stylosanthes guianensis (Aubl.) Sw. var. marginata Hassler, Repert. Spec. Nov. Reg. Veg. 16: 223. 1919. TYPE: Paraguay. Sierra de Amambay, in campis siccis Ponta Porá, Dec. 1907, E. Hassler 9938 (holotype, G!). syn. nov.

Stylosanthes gracilis Kunth var. vulgaris Burkart, Darwiniana 3 (2): 247, f. 16 d-j, pl. 15. 1939. TYPE: Paraguay. Sierra de Amambay, E. Hassler 12043 (holotype, G!). syn. nov.

Stylosanthes acuminata M. B. Ferreira \& Sousa Costa, Ann.Congr. Soc. Bot. Brasil 28: 80- 81. 1977 [1978]. TYPE: Brazil. Uberaba, BR 050, km 12 de Uberaba-Uberlandia, 1 Oct. 1976, N. M. de Sousa Costa 750 (holotype, RB!). syn. nov.

Stylosanthes grandifolia M. B. Ferreira \& Sousa Costa, Ann. Congr. Soc. Bot. Brasil 28: 84-85 1977 [1978]. TYPE: Brazil. Minas Gerais: Caeté, prox. Da cidade, M. B. Ferreira \& Sousa Costa 425 (holotype, RB!). syn. nov.

Stylosanthes aurea M. B. Ferreira \& Sousa Costa, Ann. Congr. Soc. Bot. Brasil 28: 102. 1977 [1978]. TYPE: Brazil. Diamantina, alto da Serra Miradouro da Cruz, $1100 \mathrm{~m} 18^{\circ} 15^{\prime} \mathrm{S}, 43^{\circ} 42^{\prime} \mathrm{W}^{\prime \prime}$. Sousa Costa 787 (holotype, RB!). syn. nov.

Stylosanthes guianensis (Aubl.) Sw. var. canescens M. B. Ferreira \& Sousa Costa, Empresa de Pesquisa Agropecuaria de Minas Gerais 44. 1979. TYPE: Brazil. Minas Gerais: prox. de Caeté, 4 Feb. 1976, M. B. Ferreira et Sousa Costa 308 (holotype, RB!). syn. nov.

Stylosanthes guianensis (Aubl.) Sw. var. robusta 't Mannetje, Aust. J. Bot. 25 (3): 357-358, Fig. 5-6. 1977. TYPE: Brazil. Minas Gerais: agriculture school Viscosa, 17 Feb. 1959, H. S. Irwin 2647 (holotype, NY, isotype, K!). syn. nov.

Perennial, erect, branches with whitish, curved hairs, variably dense, bristles dense, yellowish, ca. $3.5 \mathrm{~mm}$. Leaves trifoliate. Stipules 10-15 mm, basally united forming a papiraceous tube, upper half part free, linear, point tipped, with bristles, each tip with terminal bristle. Leaflets 10-27 × 2-6 $\mathrm{mm}$, narrow-elliptic, acuminate, spiny; inferior side glabrous, longitudinal veins marked, whitish green, bristles on middle vein, applied to lamina; superior side with some white hairs at leaflet base and along margins, rest glabrous, lacking bristles. Inflorescences in short spikes gobose, with 2 to 3 basal branches, each ca. 15-flowered. Bracts similar to stipules, with bristles; bracteoles 2, hyaline, ciliate, hairs red. Flowers $7 \mathrm{~mm}$, hypanthium 3-4 $\mathrm{mm}$. Calyx campanulate, $3 \mathrm{~mm}$, teeth 5, lower acute and largest, with bristles, rest rounded, all ciliated. Petals unguiculate; standard rounded, emarginated. Wings oblong; keel incurved. Fruit with single segment, $4 \mathrm{~mm}$, oblong, some thick hair son upper half, lower half glabrous. Beak short, curved, 0.5 $\mathrm{mm}$.

Distribution and habitat. This variety grows in Central and South America, from Panamá to Argentina natural pastures, savanna-like habitats, cerrados at (200) 850-1700 (4500) m a.s.l.

Additional specimens examined. ARGENTINA. Misiones: Dpto. San Pedro, San Pedro, cercanías de la ruta 14, Schwindt 4017 (LIL); Dpto. San Ignacio, Pastoreo Chico, Montes 15559 (LIL); Dpto. Candelaria, Loreto, Montes 257 (CTES); Santa Ana, Schwindt 137 (LIL); Dpto. Guarani, rte. $14 \mathrm{~km}$ 304, prox. ayo. Chajaríz, Schwindt 3189 (CTES). BOLIVIA. Santa Cruz: Sandoval, San Matías, $5 \mathrm{~km}$ SW del aeropuerto, Krapovickas et al. 36292 (CTES); Velazco, July 1892, O. Kuntze s.n. (NY). BRAZIL. In campis Selastor, Warming 3180 (C); Lagoa Santa Warming 3181 (C); Caxoeira de Campo, Martius 1122 (BR); Loefgren 5188(P). Acre: Rio Branco, E. Ule 7797 (B); Bahia: Bahia, Sellow leg. (B); Blanchet 88 (K); In campos ad Maracas, Martius s.n. (M); Mun. Ilheus, Faz. Guanabara, km 10 rd. Pontal/Olivenca, L. A. Mattos et al. 1164 (K); campus Villa Ilheus, Lungchnath 820 (K); Pr. Ilheus, Riedel 122 (K); Ilheus. Curnetto, Martius 1123 (BR). Mun. De Prado, $12 \mathrm{~km} \mathrm{~S}$ de Prado, rd. a Alcobaca, Lewis et al. $793(\mathrm{~K})$; Serra do Sincorá, Harley et al. 1740 (K). Distr. Federal: Brasilia,1819, Luschnat s.n.(P); Brasilia, Lushnat 6600 (M); Brasilia, San Carlos, Riedel 1877(P). Brasilia, Glaziou 6509 (C); Brasilia, Vale dos Buritos, H. Cavalcante de Lima et al. 260 (RB); Chapada of Antagen, ca. $10 \mathrm{~km} \mathrm{E}$ Brasilia, Irwin 11601 (P). Goiás: Goiania, estrada velha para Gaupo, cerrado, E. F. Guimarâes et al. 
309 (RB); Córrego Santa Antonio, 7 km SSE de Goiás, Kirkcbride 5521 (CTES); BR. 050, 116 $\mathrm{km} \mathrm{N}$ de Pires Belo (entre Colalao y Cristalina), Arbo et al. 3093 (CTES). Mato Grosso: BR 070, $129 \mathrm{~km} \mathrm{~W}$ de Barra do Garcas, Krapovickas et al. 43013 (CTES); $41 \mathrm{~km} \mathrm{~S}$ de Aquidauana, Faz. Varzea Alegre, Krapovickas et al. 32830 (CTES); Corrego Rancho, Mun. Araguaia, Hatschbach 34198 (CTES); E of km 261, Xavantina-Cachimbo, Philcox et al. 3357(NY); Prope Capivari, Riedel 213 (K). Mato Grosso do Sul: San Carlos, Riedel 1878(P); Caracol, 6 km la rd. p. Bela Vista, Hatschbach et al. 58855 (CTES). Minas Gerais: Feb. Martius s. n. (M); Sant Hilaire, 1816-1821, B1, 126 (P); Claussen $22(\mathrm{P})$; Caldas, Mosén 4080 (P); Diamantina, $b^{\circ}$ da Cazuza, cachoeira da Toca. Arbo et al. 5186 (CTES); Mutuca, Cerra do Corral, Roth 1909 (RB); rd. BR. 050, Tejuco, Mun. Uberlandia, Hatschbach et al. 59859 (CTES); Passagem de Mariana, Vanni et al. 513 (CTES); MG 010, 5-8 km S de Conceicao do Mato Dentro, $19^{\circ} 04^{\prime} \mathrm{S}, 4^{\circ} 26^{\prime} \mathrm{W}$, Arbo et al. 4982 (CTES); Mun. Grâo Mogol, Rio Itacambivue, Hatschbach 41267 (C); Serra Spinaco, $25 \mathrm{~km}$ E de Diamantina, near rio Jiguite, Irwin et al. 27551 (P). Pará: Spruce 266 (M). Paraná: Faz. Morungava, río do Funil, Hatschbach et al. 5325 (CTES). Piauhi: Gardner $3110(\mathrm{P})$; Espirito Santo. Conceicao da Barra, arredores, Hatschbach et al. 46992 (CTES). Rio de Janeiro: Recreio os Bandeirantes, Krapovickas et al. 23186 (CTES). Sao Paulo: Rio Claro, San Paulo, Lofgren 612(C); Itararé, Krapovickas et al. 40926 (CTES); Mun. Campinas, rd. D. Pedro p/ Magi Mirin, H. de Fleitas Leitao Filho 1787 A(K); Mun. de Butucatú, distr. Ruberâo Junior, 1-2 km SW de la Fac. de Ciências Médicas e Biología de Butucatú, 30 Gottsberger et C. J. Campos 15-301072 (RB). BRITISH GUYANA. Berbice: Courantyne, ca. $1 \mathrm{~km} \mathrm{~N}$ of creek, Pipoly 11574 (NY). Rupununi: Northern Savanna, Takutu, 2 miles S of St. Ignatius, 350 ft. s. m.10-11Aug. 1963, Goodland et al. 360 (NY). 3 mi. S of Mentiziero, Goodland 5539 (NY); Karanambo, Maas 7237 (CTES, NY). COSTA RICA. Cartao: Strabe, 226 km von Errichen des Ortes Loaiza, Dolbeler 3400 (M). San José: Mora Colón, camino a Piedras

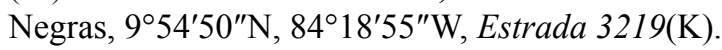
FRENCH GUIANA. Cayenne: Cedex, savanne Yiyi, long. $53^{\circ} 2^{\prime}$ lat. $5^{\circ} 24^{\prime}$, Tonola Marlot D. 282(K): Guyana angl. Schombrurg 390 (P). GUATEMALA.
Santa Rosa: Cerro Redondo, Hayde at Lux 6120 (K). PANAMA. Colón: Canal zone, $1 / 2 \mathrm{~km} \mathrm{~W}$ of Summit Gardens, Nee 9494 (CTES). PARAGUAY. Amambay: P. N. Cerro Corá, Soria et al. 2051 (CTES); 65 km W de P. J. Caballero, Krapovickas et al. 14184 (CTES). Central: Jardín Botánico de Asunción, Schinini 1350 (CTES); San Lorenzo, Capilla del Monte, Bordas 3642 (CTES). Guairá: Col. Independencia, $56^{\circ} 17^{\prime} \mathrm{W}, 25^{\circ} 57^{\prime} \mathrm{S}$, ayo. Guazú, camino a San Gervasio, Schinini \& Vanni 28078 (CTES); Caaguazú, 14 km N de Caaguazú, camino a Yhú, Schinini \& Vanni 28095 (CTES); Caazapá. Santa Ursula, $55 \mathrm{~km}$ NE de Yuty, Schinini \& Vanni27814 (CTES). Itapúa: Rte. 6, $5 \mathrm{~km} \mathrm{~N}$ de Artigas, Schinini \& Vanni27673 (CTES); Col. Gral. Belgrano, E San Miguelito, T. M. Pedersen 3263 (Herb. Pedersen, CTES). Misiones: E La Soledad, $3 \mathrm{~km} \mathrm{~S}$ de Santiago, Schinini \& Vanni 26103 (CTES). Paraguarí: $10 \mathrm{~km}$ de Paraguarí, camino a Peribebuy, Krapovickas et al. 12427 (CTES). San Pedro: In campo pr. San Estanislao, Hassler 6008(G!); sintipos de Stylosanthes guianensis var. genuina Hassl. $10 \mathrm{~km} \mathrm{~W} \mathrm{de} \mathrm{San} \mathrm{Estanislao,}$ bañado del río Tapiracuí, Krapovickas et al. 44879 (CTES). PERU. Cuzco: La Convención. AobambaHuadquiña, Vargas 8162 (CTES). VENEZUELA. Bolivar: Curran 191 (NY); Canaima, Merxmüller 22959 (M). Trujillo: Near Escuque, Truj. rute to Valera, Pittier 13150 (NY).

Obs. Based on the studied type material and the following examined specimens (cited by't Mannetje,1977), E. Hassler 7781 (G), Morong $399 b$ (NY) and Pedersen 3263 (CTES), it is impossible to distinguish $S$. guianensisvar. robusta from $S$. guianensis var. gracilis. In addition, Stylosanthes acuminata can not be differentiated from S. guianensis var. gracilis; the characteristics of the former are found within the variation of the latter, which is widespread in the Americas. The type specimen of $S$. acuminata is similar to that of $S$. guianensis var. gracilis, even though its fruits are slightly smaller (3-3.5 mm). Similarly, it is impossible to distinguish $S$. guianensis var. canescens from $S$. guianensis var. gracilis.

In the study by Calles et al. (2010), the name Stylosanthes gracilis is considered as a separated species. However, their study includes problems with typification and evaluation of the varieties. The fruit is a diagnostic feature in Stylosanthes, but 
in this case it is impossible to distinguish the two species based on fruits. Therefore, $S$. gracilis is here considered as a variety of $S$. guianensis.

5. Stylosanthes hamata (L.) Taubert, Verh. Bot. Vereins Prov. Brandenburg 32 (1): 22. 1890. Hedysarum hamatum L., Syst. Nat. (ed. 10), 2: 1170. 1759. TYPE: Jamaica. Sloane, Voy. t. 119, fig. 2. 1707 (holotype, BM, Herb. Sloane, photo BM 000589680!).

Perennial, prostrate. Branches with whitish, thin, weak hairs. Stipules $7 \mathrm{~mm}$, united, point tipped apically, $5 \mathrm{~mm}$. Petioles $5 \mathrm{~mm}$. Leaflets 15 $\mathrm{mm} \times 3 \mathrm{~mm}$, elliptic, inferior side with marked, whitish vein sand white, dense, appressed hairs, bristles along margin, superior side glabrous. Inflorescences globose, spikes, basally branched, 5- to 7-flowered; bracts similar to stipules, $5 \mathrm{~mm}$, hyaline, with bristles. Flowers $5 \mathrm{~mm}$. Calyx with 5 teeth, inferior largest, $2 \mathrm{~mm}$. Fruit with 2 segments, superior $7 \mathrm{~mm}$, inferior $2 \mathrm{~mm}$, pubescent. Beak slightly incurved.

Distribution and habitat. It inhabits primarily throughout the Caribbean and the southern United States, Mexico and Colombia in natural pastures savanna-like habitats.

Additional specimens examined. BRITISH VIRGIN ISLANDS. Overse as territories from airport to the Settlement, Pollard 1234 (K). COLOMBIA. Atlantico: Salgar, cerca del Pto. De Colombia, E. Yumis de Katt'ah et al. 19 (SI). COMMON WEALTH OF THE BAHAMAS. Isla Fortuna, Eggers 3821 (K). CUBA. Champs de la Havana, Galeotti 3401-Cuba MIO(BR). HAITI. Vic. of Porto de Paix, C.M. Leonard et Leonar $1100(\mathrm{~K})$. JAMAICA. Santo Tomás: Eggers 105 (K, M); Eggers 18050(M); Pera Beach, E-TRobertson 2798 (K). MÉXICO. Jalisco: C. A. Purpus 4245 (C). PUERTORICO. Antigua San Johns lensing Wullschlägel 141 (BR); Rio Juez, Coamo, I. Urban 3280 (B); Bayamon, in Litoralis ae "Polo seco," I. Urban 1092 (B); Porto Rico. Bertero s.n. (M). REPÚBLICA DOMINICANA. Santo Domingo: Los Ríos., Bouharmont 18504 (BR); Monte Cristyon río Mao, Valeur 802(K). UNITED STATES. Florida: Monroe Co. Big. Pine key. Along old route NE of Inn, E. P. Killip 40807
(SI). WEST INDIES. Lesser Antillis exploration anegads rocky plant near Settlement, N. L. Britton et al. $1007(\mathrm{~K})$.

Obs. The type locality of $S$. hamata is Jamaica. Given its use as a forage plant, the species is currently distributed from the southern United States, across the Caribbean to northern South America, being cultivated in Colombia, Brazil and Paraguay. I examined the following specimens that Taubert used to suggest the new combination: Wullschlägel 141 (BR), Bertero s.n. (M), and Eggers 105 (M).

6. Stylosanthes hispida Richard, Actes Soc. Hist. Nat. Paris 1: 112. 1792. TYPE: French Guiana, E Cayenne, missarum a Dominico, Leblond (holotype, P, not seen). Fig. 6.

Stylosanthes cayennensis Mohlenbr., Ann. Missouri Bot. Gard. 44 (4): 337-338. 1958. TYPE: French Guiana, Vic. of Cayenne, 25 July 1921, W. E. Broadway 972 (holotype, GH not seen, isotype, $\mathrm{K}$ !). syn. nov.

Perennial, with numerous branches, indument dense with weak, whitish hairs, appressed to surface, without bristles. Leaves trifoliate, pinnatetrifoliate. Stipules united to one another and with petiole basally, free in upper third, apically point-tipped, with bristles up to $4 \mathrm{~mm}$. Leaflets $8,1-5 \times 12 \mathrm{~mm}$, narrow-elliptic, with apical bristle; inferior side with conspicuous veins, with weak, appressed hairs, without bristles, superior side glabrous. Inflorescences terminal in globose spikes, 15 or more flowers. Bracts with 3 aristae, involving flowers, with bristles up to $4 \mathrm{~mm}$, on superior part and one aristae, giving hispid aspect to inflorescence, from which derived the species epithet. Flowers $3 \mathrm{~mm}$. Hypanthium $5 \mathrm{~mm}$. Fruit with 2 glabrous segments, each segment $3 \mathrm{~mm}$, with a marked reticulation. Beak short, strongly curved.

Distribution and habitat. This species is found in French Guiana and northern Brazil in natural pastures, savanna-like habitats and cerrado, at 500$700 \mathrm{~m}$ a.s.1.

Additional specimens examined. BRAZIL. Amapá: Iparapé do Praia, rd. to Amapá, Murca Pires et al. 52237 (K, NY); Territorio Amapá, 
Bol. Soc. Argent. Bot. 52 (3) 2017

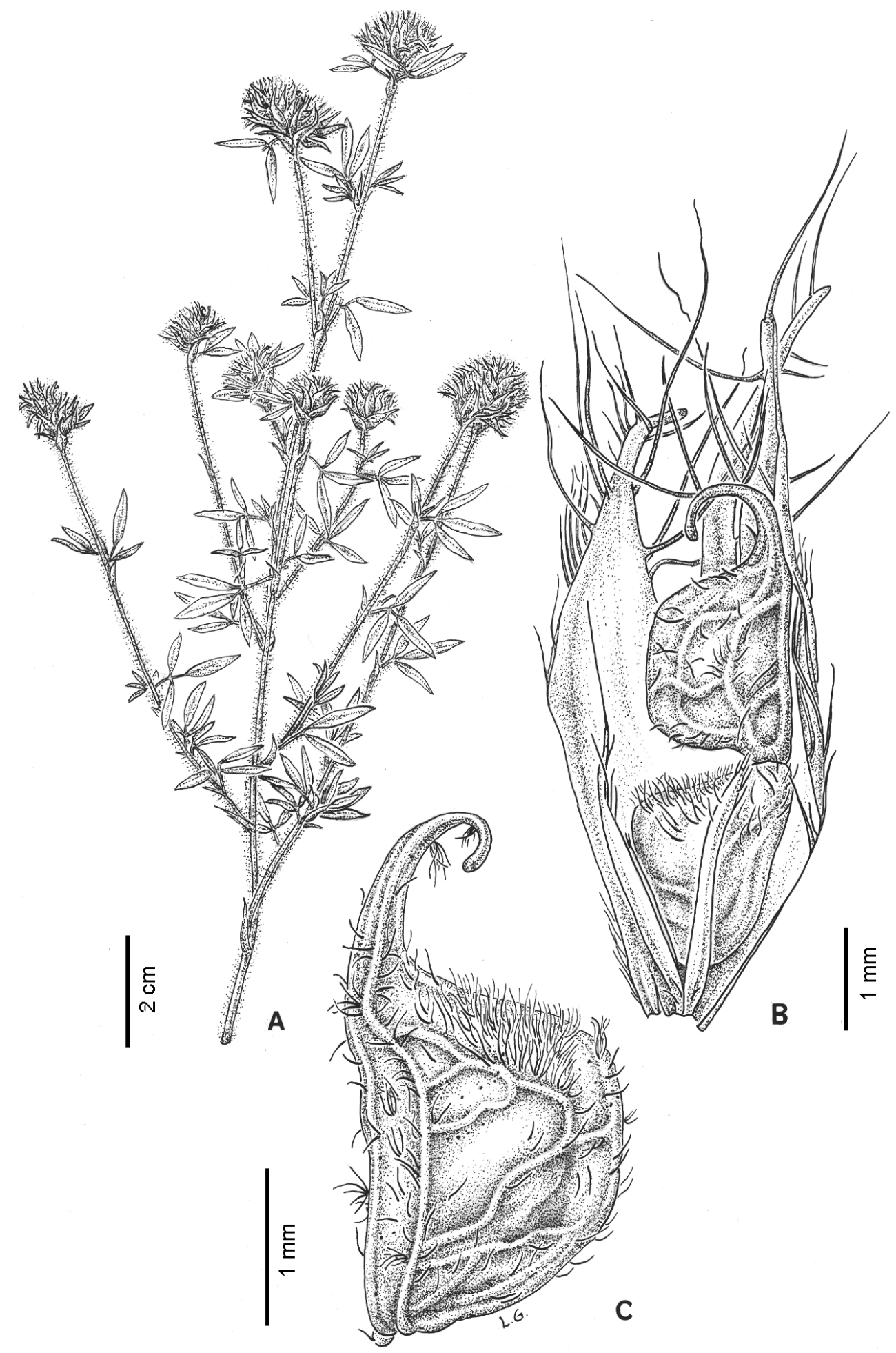

Fig. 6. Stylosanthes hispida. A. Plant. B. Fruit. C. Detail of the superior segment. (Pire \& Cavalcante 52489). 
rio Flechal, Pires \& Cavalcante 52489 (NY); rio Oiapoque, J: M. Pires 47536 (B); Rio Oyapock, Rocbe Monfere, Luetzelburg 20280 (M). Pará: 2 $\mathrm{km}$ W of AMZA, Serra dos Carajás, Sperling et al. 5648 (NY). FRENCH GUIANA. Región TumucHumac, fronteire Brasil, G. Francoise, Mitaraka $\mathrm{S}, 35 \mathrm{~km}$ W-SW du sommet, J. De Granville 1208 (NY); Mitaraka nord, $1.5 \mathrm{~km} \mathrm{~N} \mathrm{du} \mathrm{sommet,}$ J. De Granville 1177 (NY); Zuid River, $40 \mathrm{~km}$ along confluence with Lucie River, Irwin et al. 57580 (NY); Cayenne. Massif des Emerillons, versant Nord, J. De Granville 3928 (NY); Dégrand Saramaca Bassim du Kourou, Cremers \& al. 10647 (BR); Roche Koutou Bassin de Haut-Marquini, 2 $\mathrm{km}$ au nord Granville et al. 9297 (B).

Obs. This species was considered a synonym of S. guianensis by Mohlenbrock (1957) and 't Mannetje (1977). Based on analysis of the original description and of specimens from various countries and herbaria, I concluded that $S$. hispida is an independent species from $S$. guianensis. In $S$. hispida, the fruit has two segments and is glabrous or with a few weak hairs being completely different from the fruit of $S$. guianensis, which has only one segment with thick and succulent hairs.

7. Stylosanthes hippocampoides Mohlenbr., Ann. Missouri Bot. Gard. 44 (4): 339, fig. 7. 1958. TYPE: Argentina, Córdoba, Sierra de Achala, Kurtz 8303 (holotype, NY, not seen, isotype, R!). Fig. 3.

Stylosanthes guianensis (Aubl.) Sw. var. intermedia (Vogel) Hassler, Repert. Spec. Nov. Reg. Veg. 16: 221. 1919.

Stylosanthes montevidensis var. intermedia Vogel, Linnaea 12: 67. 1838. TYPE: Paraguay. Ad margine silvae Cord. de Altos, E. Hassler 1803 (lectotype designated here, G!).

Stylosanthes gracilis Kunth var. subviscosa (Benth. ex Mart.) Burkart, Darwininana 3: 248. 1939.

Perennial, branches prostrate, with white weak hairs and very dense bristles, $4 \mathrm{~mm}$. Leaves trifoliate. Stipules 6-10 mm, united at leaf base, papiraceous, point tipped, with bristles. Leaflets 10 $17 \times 3-3.5 \mathrm{~mm}$, narrow-elliptic, mucronate, inferior side glabrous, with bristles on veins and margins, superior side totally glabrous, without bristles. Inflorescences terminal, not branched, spikes, globose densely flowered. Bracts similar to stipules, with very dense bristles. 2 hyaline bracteoles at base of each flower, ciliate. Flowers $6 \mathrm{~mm}$. Calyx $3 \mathrm{~mm}$, campanulate, teeth acute, ciliate. Petals unguiculate. Standard rounded. Wings oblong. Fruit with only 1 segment, $4.5 \mathrm{~mm}$, oblong, nervature marked, glabrous, some glandular hairs on upper half. Beak hook-like, spiral, $1 \mathrm{~mm}$.

Distribution and habitat. It dwells in Bolivia, Paraguay, Argentina, Uruguay and western and southern Brazil, in natural pastures, savannalike habitats and cerrado at 550-1200 m a.s.l. In Argentina, the Province of San Luis represents the southern limit of the geographic distribution of $S$. hippocampoides.

Additional specimens examined. ARGENTINA. Chaco: Dpto. $1^{\circ}$ de Mayo, Colonia Benítez, Schulz 11641 (CTES); Mayor L. J. Fontana, Schulz 1151 (CTES, SI). Córdoba: Dpto. Santa María, Villa Carlos Paz, A. de la Sota 1634 (LIL); Dpto. Río Segundo, Río Segundo, Krapovickas et al. 7572 (CTES); Sierra Chica, E La Reducción, Burkart 7391 (SI); Sierra de Achala, N de la cuesta de Copina, Hieronymus 653 (SI); Dpto. Calamuchita, Altos Pampa, A. Krapovickas 7765 (CTES). Entre Santa Rosa y Soconcho (Sierra Chica), 22 Jan. 1952, Krapovickas 7654 (CTES); Dpto. Colón, E La Serrana - El Cuadrado, Luti 7282 (CTES); Rio Ceballos, Villa Carenzo 3588 (LIL); Sierra Chica, entre los Saltos y el Cuadrado, camino Río Ceballos, a la Falda, Krapovickas 7546(K). Corrientes: Dpto. Ituzaingó, 10 km W de Ituzaingó, rte. 12, Krapovickas 7896 (LIL); Dpto. Santo Tomé, Cuay Grande, Ibarrola 1607 (NY); Dpto.San Martín, Colonia C. Pellegrini, $R$. Spegazzini 1078 (CTES); Tres Cerros, entre La Cruz y Yapeyú, Burkart 8168 (SI); San Martín, Ibarrola1683 (NY); Dpto. Mburucuyá, E Santa Teresa, Carnevali 5975 (CTES); idem, T. M. Pedersen 79 (NY); Dpto. Gral. Paz, ca. 15 km S de Gral. Paz, Krapovickas et al. 7851 (CTES); Itá Ibaté, ayo. Santa Lucía, Krapovickas 7881 (LIL); Dpto. Capital, ayo. Riachuelo y rte. Nac. 12, Vanni 175 (CTES); Santa Catalina, Ibarrola 928 (NY); Dpto. Paso de los Libres, Bonpland, costa del río Uruguay, Ibarrola 2142 (NY); Dpto. Monte Caseros, cercanías de Juan Pujol, Ibarrola 2331 (LIL). Entre Ríos: Dpto. Colón, Parque Nac. 
El Palmar, ayo. Los Lores, Cusato 499 (CTES); Colón, T. Meyer 10604 (LIL); Dpto. Federación, Santa Ana, barranca del río Uruguay, Burkart et al. 29336 (CTES); Dpto. Concordia, Concordia, I-1936 Faquahrson, Herb. de Burkart 7797 (SI). Formosa: Dpto. Patiño, E Muchutti, $5 \mathrm{~km} \mathrm{~N}$ de las Lomitas, Fortunato \& al. 3394 (CTES). Misiones: Dpto. Santa Ana, Santa Ana, Rodriguez 756 (SI); Dpto. Candelaria, Loreto, J. E. Montes 11239 (CTES); idem Est. Experimental, Krapovickas 7916 (LIL). San Luis: Merlo, Piedra Blanca, Burkart 13913 (SI). BOLIVIA. Santa Cruz: Cerro San Miguel $19^{\circ} 18^{\prime} \mathrm{S} 060^{\circ} 39^{\prime} \mathrm{W}$, Ramella et al. 6860 (CTES, G). BRAZIL. Goiás: Prov. De Goias, Glaziou 20919 (K); Cerrado, $9 \mathrm{~km} \mathrm{~S}$ of Guará, Irwin et al. 21515 (NY). Mato Grosso: Mun. De Xavantina, Onishi et al. 980 (K). Rio Grande do Sul: Sto. Angelo, B. Rambo 53030(B); BR 101, km 6 Campo Bonito, Krapovickas et al. 38527 (CTES); Mun. Rosario do Sul, Rosario do Sul, río Sta. María, Krapovickas et al. 22803 (CTES); km 223 rte. Sta. María a Sao Borja, $70 \mathrm{~km}$ SE de S. Borja, Krapovickas et al. 38384 (CTES). PARAGUAY. Caaguazú: 5 km E de Caaguazú, Rte. 2, 8 Krapovickas et al. 12490 (CTES). Central: Itá Granja Isapy, orilla del ayo. Lazarillo, Krapovickas et al. 12124 (CTES); Asunción, B ${ }^{\circ}$ San Vicente, Schinini 7957 (CTES); Near Itaguá, Stevens et al. 94 (NY). Concepción: Zurichen rio Apa und rio Aquidaban, Fiebrig 4397 (K). Cordillera: In campo pr. Cord. de Altos, Hassler 1584 (G); "In campo pr. Itacurundú", Hassler 1570 (G); In campo, pr. San Tomás, Hassler 1688 (G); Cordillera de Altos, Fiebrig 405 (K); 17 km de Paraguarí, camino a Piribebuy, Krapovickas et al. 12454 (CTES); Caacupé, B ${ }^{\circ}$ Kennedy, Bordas 4061 (CTES). Itapúa: Isla Yacyretá, $56^{\circ} 47^{\prime} \mathrm{W}$,

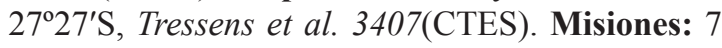
km E de San Ignacio, camino a Santa Rosa, Arbo et al.6118 (CTES). Ñeembucú: Pilar, Schulz 7882 (CTES). Presidente Hayes: Cerrito, Misión San Francisco, 5 km de Benjamín Aceval, Arenas 1018 (CTES).

Obs. Several problems were found with the synonymy of this taxon, mainly due to diverse interpretations by different authors. Burkart (1967) was the first to use S. hippocampoides, and considered $S$. gracilis var. subviscosa as a synonym of this species. Later, he used the synonym to circumscribe a number of Argentinean specimens deposited at SI (Burkart 1939), which in fact correspond to S. hippocampoides. Mohlenbrock and 't Mannetje (1977) considered S. gracilis var. subviscosa as a synonym of $S$. guianensis. 't Mannetje (1977) considered S. gracilis var. subviscosa as a synonym of $S$. guianensis var. intermedia. All specimens cited by Hassler (1919) as $S$. guianensis var. intermedia correspond to $S$. hippocampoides. This identification error by 't Mannetje is also true for the cultivar cv. Oxley, commercialized for Australia, which actually belongs to $S$. hippocampoides.

8. Stylosanthes humilis Kunth, Nov. Gen. Sp. 6: 506. 1824. TYPE: Venezuela. Creciet to Orinocum, prope Carichaná, Humb. et Bonpl. s.n. (holotype, $\mathrm{P} !)$.

Perennial, branches prostrate, little developed. Branches and rest of plant with bristles up to 4 $\mathrm{mm}$; hairs whitish, weak, incurved. Stipules 9-10 $\mathrm{mm}$, united at base and point-tipped atapex, with bristles. Leaflets $11 \times 3 \mathrm{~mm}$, elliptic, mucronate, inferior side with bristles and gland dots, superior side with applied hairs, bristles long leaflet margin. Inflorescences elongate, terminal oraxillary, 19-flowered, not branched. Bracts similar to stipules, with bristles. Flowers $6 \mathrm{~mm}$. Fruit with 2 segments, inferior with dense yellowish hairs, 2 $\mathrm{mm}$, superior with sparsely isolated hairs, $7 \mathrm{~mm}$. Beak $4 \mathrm{~mm}$, almost equal or larger than segment, very elongated and slightly curved apically.

Distribution and habitat. It lives in Mexico, Costa Rica, Bolivia and Brazil in natural pastures, savanna-like habitats, and cerrado.

Additional specimens examined. BOLIVIA. Santa Cruz: prov. Chiquitos, $3 \mathrm{~km}$ de Roboré, Krapovickas et al. 36400 (CTES); 3 km W de San José, camino a EL Tinto, Dematteis et al. 2291 (CTES). BRAZIL. Bahia: $15 \mathrm{~km}$ W de Joazeiro, Krapovickas et al. 12924 (CTES). Mato Grosso: Torixoreu, $20 \mathrm{~km} \mathrm{~S}$, Hatschbach 34183 (CTES). Matto Grosso do Sul: Mun. Porto Murtinho, rd. Bonito-Campo dos Indios, río Aquidaban, Hatschbach et al. 74224 (CTES, MBM); Mun. Corumba, Faz. São Vicente, Nhecolandia, A. Pott 2066 (CTES). COSTA RICA. Alojuela: Orotina, Coyolar, Estrada \& al. 3118 (K). MÉXICO. 
Chiapas: Mun. Simojovel, rute from El Bosque Simojobel, 3000 ft., Shilom 3096(CTES). Cutzamala: Distr. Coyuca, G.B. Hinton 6985 (K). PARAGUAY. Boqueron: Col. Neuland, escapada de cultivo, Vanni et al. 2086 (CTES). SURINAM. Saramacca: Exp. Farm Coebiti, Everaarats 959 (CTES). VENEZUELA. Apure: $21 \mathrm{~km} \mathrm{NE}$ of Mantecal, G. Davidse 3890 (CTES, MO).

Obs. Stylosanthes humilis is a native species of Venezuela that has been introduced in many countries as a forage plant, including Brazil, Bolivia and Paraguay.

9. Stylosanthes leiocarpa Vogel, Linnaea 12: 64-65. 1838. TYPE: Uruguay: Hbt. in Brasilia; in Montevideo s. d., F. Sellow s. n. (holotype, BHU, photo 2245, B!, isotype, K!). Fig. 3.

Perennial, branches decumbent to erect, indument throughout with yellow bristles and weak whitish hairs. Leaves pinnate-trifoliate. Stipules united, papiraceous, accompanying stem, free at petiole insertion, $5 \mathrm{~mm}$, filiforms, with bristles. Petioles $5 \mathrm{~mm}$. Leaflets $7-8 \times 3 \mathrm{~mm}$, elliptic, mucronate, lateral ones asymmetric, inferior side with bristles with thickened reddish base along margins, white weak hairs appressed or not to surface; superior side with few bristles and few gland dots, glandular hairs. Spikes globose, widened, up to 8-flowered, without ramifications at base. Flowers $13 \mathrm{~mm}$. Hypanthium $6 \mathrm{~mm}$. Calyx $5 \mathrm{~mm}$, campanulate, teeth 5 , apically rounded. Petals unguiculate. Standard 8 $\mathrm{mm}$, rounded. Wings oblong. Keel incurved. Fruit with 2 segments, glabrous. Bracteoles 2, basal, hyaline. Inferior segment $3.5 \times 2 \mathrm{~mm}$, superior with prolonged beak. Beak slightly incurved, $3 \mathrm{~mm}$, hairs sparse at base and along internal side of beak curvature, segment body $3 \mathrm{~mm}$.

Distribution and habitat. This species lives in northeastern and southern Brazil, eastern Paraguay, northeastern Argentina, and Uruguay, in field sand lomadas with sandy soil.

Additional specimens examined. ARGENTINA. Corrientes: Dpto. San Roque, Rte. 123, 5 km E empalme Rta. 12, camino a Mercedes, Vanni 113 (CTES); Dpto. Bella Vista, 7 km E de Bella Vista, Battú 32 (CTES); idem Quarín 3082 (CTES); Dpto.
Goya, 20 km SE de Goya, Carnevali 1922 (CTES). BRAZIL. Bahia: Margen del río Una, Belén et al.1019(NY); rio Paraguacu, Hatschbach 45110(K); Schultze-Kraft CIAT 10097(K); Rio Grande do Sul. Est. De Silvicultura. Sta María, Camargo 19 (B); 12 km E de Canela, camino a Sao Francisco de Paula, Krapovickas et al. 44684 (CTES); Mun. Rosario do Sul, $14 \mathrm{~km} \mathrm{~W}$ de Rosario do Sul, BR 290, Krapovickas et al. 22791 (CTES); Torres, Rambo 56467 (B); Vila Nova, pr. Porto Alegre, Rambo 40675 (CTES); Porto Alegre, Canôas, Palacios y Cuezzo 316 (CTES); $55 \mathrm{~km}$ de Sao Gabriel, camino a Bage, Krapovickas et al. 22850 (CTES); Torres, Krapovickas et al. 37699 (CTES); Lagoa dos Barros, p. Osorio, Rambo 44569 (CTES); Cassino, T. M. Pedersen 6674 (Herb. de Pedersen, CTES); T. M. Pedersen 9380 (C). PARAGUAY. Amambay: E Santa Teresa, potrero Nagoa, Soria 4908 (CTES). Concepción: $22 \mathrm{~km}$ NE de Concepción, Hacker 1042 (CTES). Paraguarí: Paraguarí, Sparre y Vervoost 595 (CTES). URUGUAY. Montevideo: Pocitos, Carrasco, Herter 65 (K, NY); Pocitos, Herter 76691 (CTES, M); San José, Barra, Herter 65 (B).

Obs. This represents the first report of $S$. leiocarpa in Argentina, where it occurs in the Province of Corrientes, being rather uncommon.

10. Stylosanthes linearifolia M. B. Ferreira \& Sousa Costa, Anais Soc. Bot. Brasil 28: 86-87. 1977 [1978]. TYPE: Brazil. Minas Gerais: Mun. de Uberaba, BR 050, Uberaba. Uberlandia, km 12 alt. 850 m. $19^{\circ} 45^{\prime}$ S, $47^{\circ} 55^{\prime} \mathrm{W}, 1-\mathrm{X}-1976$, N. M. Sousa 753 (holotype, RB!). Fig. 7.

Perennial, up to $50 \mathrm{~cm}$. Branches suberect; indument with sparse, small, curved, white hairs, and scattered bristles. Leaves digitate-trifoliate. Stipules $8 \mathrm{~mm}$, united, with bristles, ending in 2 aristae with anapical bristle. Leaflets linear, 15-24 $\times$ 1-1.5 mm, inferior side glabrous, with bristles and red gland dots, superior side glabrous, bristles abundant along leaflet margins. Inflorescences, spikes globose, 3-to5-flowered. Bracts similar to stipules, open, with yellow bristles up to $2 \mathrm{~mm}$. Flowers $5 \mathrm{~mm}$. Hypanthium $3 \mathrm{~mm}$. Calyx $2 \mathrm{~mm}$. Petals unguiculate. Standard $5 \times 3 \mathrm{~mm}$, oblong. Wings $4 \times 1 \mathrm{~mm}$, oblong. Fruit with 1 segment, oblong, cuneate, castaneous at maturity, $3 \times 2 \mathrm{~mm}$, 
Bol. Soc. Argent. Bot. 52 (3) 2017

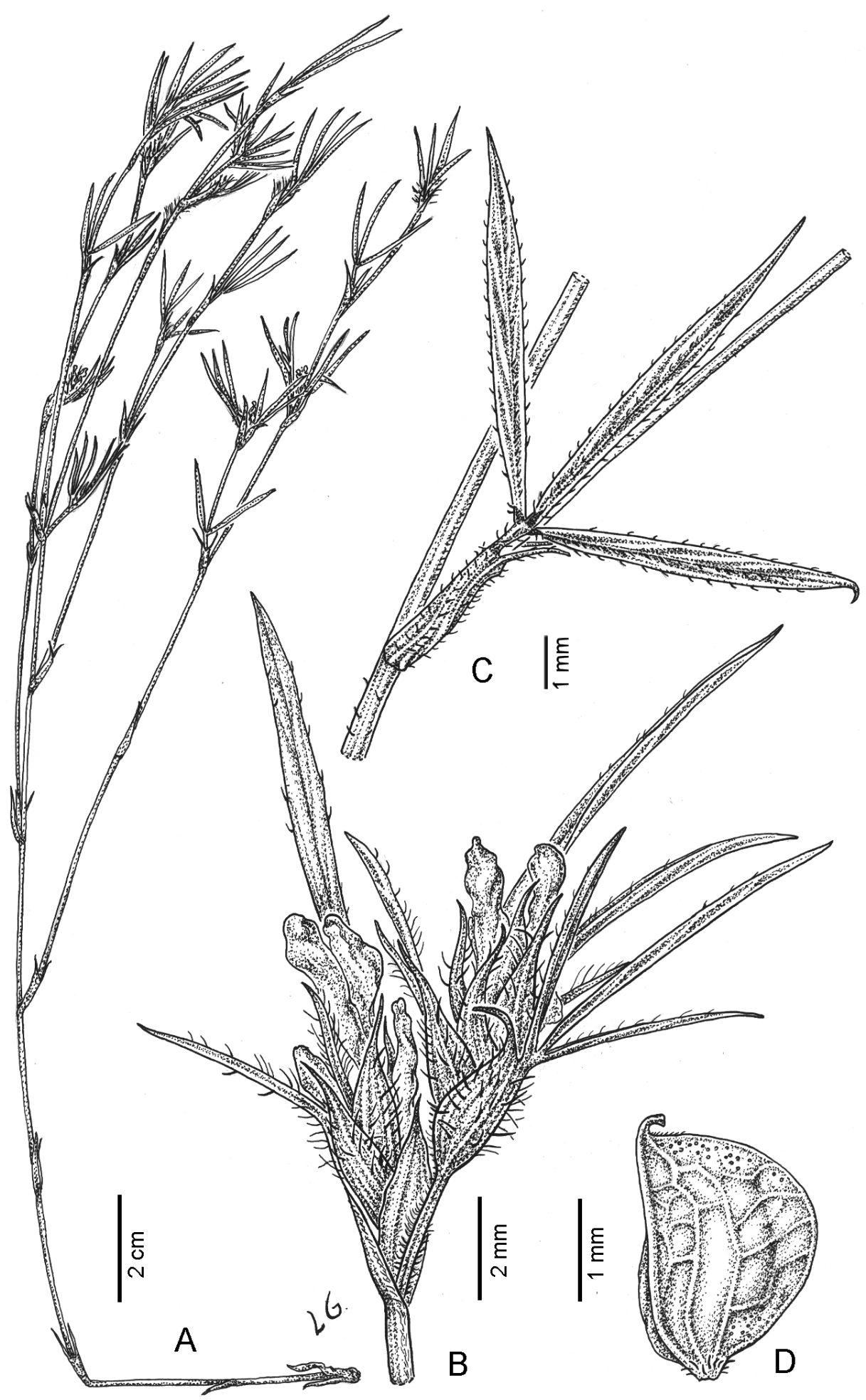

Fig.7. Stylosanthes linearifolia. A. Plant. B. Inflorescence. C. Leaf. D. Fruit. (Herb. 67377 R). 
some thick hairs on upper part of segment. Beak 0.2 $\mathrm{mm}$, hook-like.

Distribution and habitat. It is found in Brazil in natural pastures, savanna-like habitats, and cerrado.

Additional specimens examined. BRAZIL. Paraná: Capao Grande, Dusén 3982 (R).

11. Stylosanthes longiseta Micheli, Mém. Soc. Phys. Genève 28 (7): 18 tab. IV. 1883. TYPE: Paraguay. In campis prope Caaguazú, Jan., Balansa 1842 (holotype, G, not seen, isotype, P!). Fig. 8.

Stylosanthes guianensis (Aubl.) Sw. subsp. anomala Hassler var. longiseta (Micheli) Hassler, Repert. Spec. Nov. Reg. Veg. 16: 221. 1919.

Perennial, rhizomatous, erect, ca. $35 \mathrm{~cm}$ high. Stems with dense indument of weak, incurved hairs and dense bristles up to $5 \mathrm{~mm}$. Leaves trifoliate. Stipules united to one another and to petiole base, forming a tube, with 2 lateral aristae, bristles throughout. Leaflets $14-30 \times 9-13 \mathrm{~mm}$, obovate, inferior side with setiform emergences on median vein and lamina, marginal veins prominent, greenyellowish, without bristles and red gland dots; superior side glabrous, with bristles and some hairs on principal vein. Spikes globose 26-flowered. Bracts $5 \mathrm{~mm}$, basally broadened and papiraceous, with 2 lateral aristae and terminal lamina similar to leaflet, with bristles throughout. Flowers $7 \mathrm{~mm}$. Hypanthium $4 \mathrm{~mm}$. Calyx $3 \mathrm{~mm}$, campanulate, teeth 5 , inferior largest, all ciliate. Standard $7 \mathrm{~mm}$. Fruit with 2 hyaline bracteoles, ciliate, 1 segment 4 $\times 3 \mathrm{~mm}$, rounded, covered with capitate hairs. Beak very short, $0.5 \mathrm{~mm}$, hooked.

Distribution and habitat. Stylosanthes longiseta is found in central and eastern Paraguay in sandy soils, apparently associated with "palmares" vegetation dominated by Butia paraguayensis (Barb. Rodr.) L. H. Bailey.

Additional specimens examined. PARAGUAY. "Paraguay, In regione fluminis Carimbatay, September", E. Hassler 4573 (G!). Caaguazú: $32 \mathrm{~km} \mathrm{~N}$ de Caaguazú, camino a Yhú $22^{\circ} 10^{\prime} \mathrm{S}$, $55^{\circ} 58^{\prime} \mathrm{W}$, Krapovickas et al. 45764 (CTES); Regiones fluminis Yhú, Hassler 9533 (P); 8 km S de Yhú, Schinini 23014 (CTES). Canendiyú:
Reserva Natural del Bosque Mbaracayú, Horqueta mí. Precordillera de la sierra de Mbaracayú. $35 \mathrm{~km}$ del puesto Yeyuí, Schinini et al. 33326 (CTES).

12. Stylosanthes macrosoma S. F. Blake, Proc. Biol. Soc. Washington 33 (9): 52. 1920. TYPE: Sin loc. "central Paraguay", 1888-1890, T. Morong 255 (holotype US, isotype, K!, NY!). Fig. 3.

Perennial. Branches erect to suberect, green, with indument of whitish, weak, incurved hairs, variable in density, generally denser towards apex; plant covered throughout with thickened bristles, basally reddish and apically white-yellowish up to $1.5-2 \mathrm{~mm}$ long. Leaves trifoliate. Leaflets $11-$ $19 \times 1.5-4 \mathrm{~mm}$, terminal largest, narrowly elliptic, cuneate at base; inferior side with strongly marked veins, some small appressed hairs, longer on margins, and bristles on veins; superior side with gland dots, with same indument as the anterior side. Stipules united, prolonged in 2 aristae, 5-6 $\mathrm{mm}$, on both sides of petiole, generally ending in a seta. Petioles 3-6 mm long. Inflorescences in spikes globose, up to 7 flowers. Bracts similar to stipules, but with 1 terminal leaflet, 2 bracteoles, hyaline, ciliate. Flowers, $6 \mathrm{~mm}$. Hypanthium $5.5 \mathrm{~mm}$ long. Calyx $2.5 \mathrm{~mm}$ long, 5 lobes obtuse, ciliate, 2 superior ones united. Corolla petals, unguiculate, the standard rounded, slightly emarginate, $6 \mathrm{~mm}$ long. The wings slightly incurved, oblong, with a conspicuous nectary between claw and lamina. The keel incurved, adhering completely to androecial tube. Fruit with only 1 fertile segment $8-9 \mathrm{~mm}$, with prominent venation, glabrous, hairs hirsute only along inner side of beak; the beak spiral.

Iconografy. Burkart (1939): Fig. 15 g, n.

Distribution and habitat. It grows in Argentina and Paraguay in savanna-like habitats.

Additional specimens examined. ARGENTINA. Chaco: Dpto. $1^{\circ}$ de Mayo, Colonia Benítez, Schulz 16492 (CTES); Dpto. 9 de Julio, Las Breñas, Schulz 10574 (CTES). Corrientes: Dpto. Capital, ayo. Riachuelo y rta. 12, Krapovickas et al. 13574 (CTES); 16 km ENE de Corrientes, rta. 12, E Las Mercedes, Quarín 3256 (CTES); Molina Punta, A. Krapovickas et al. 11753 (CTES); idem Vanni 436 (CTES); Dpto. San Cosme, 4 km E de Paso 


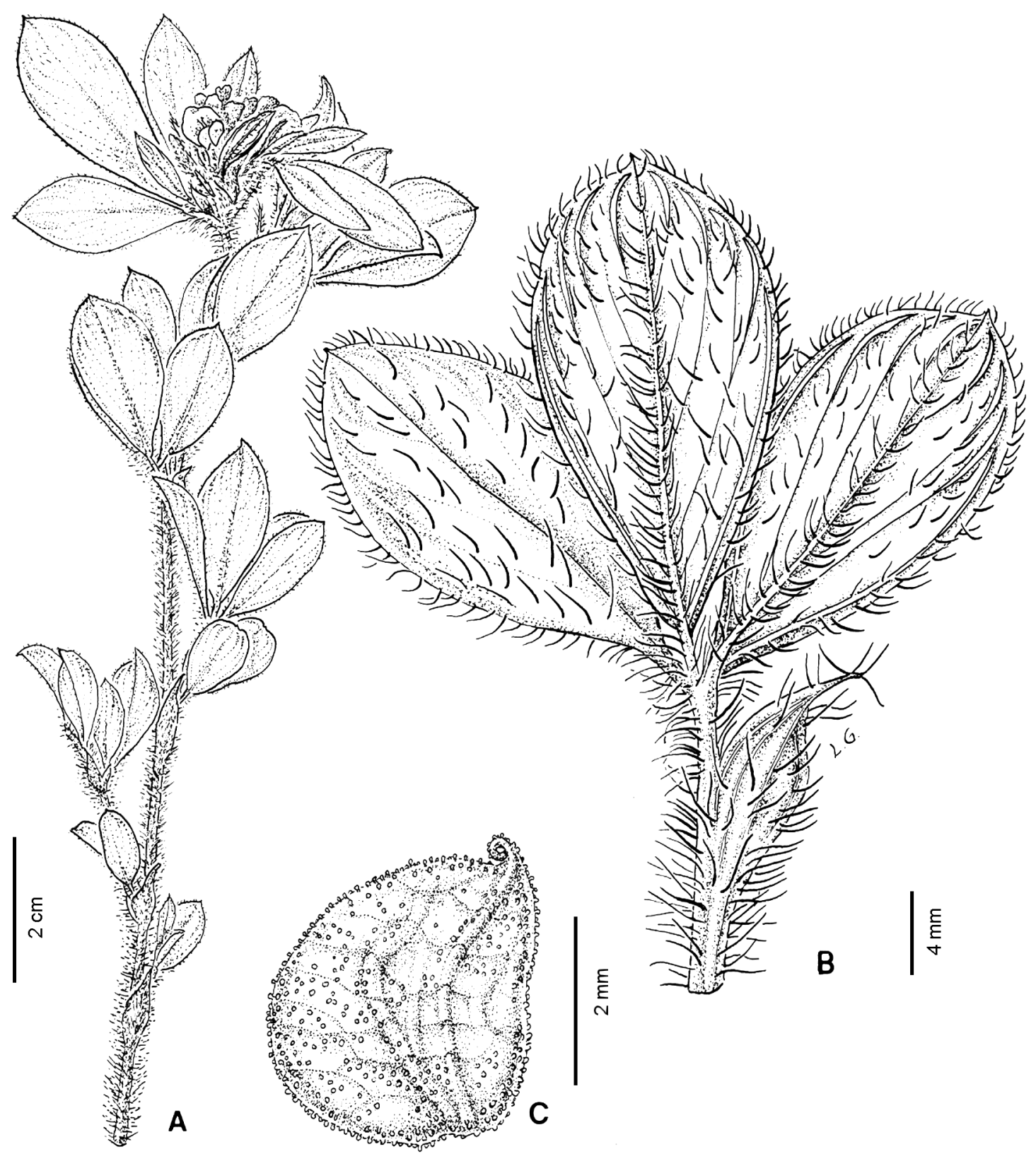

Fig. 8. Stylosanthes longiseta. A. Plant. B. Leaf with stipules. C. Fruit. (Hassler 9532, except fruit Schinini 23014).

de la Patria, Krapovickas et al. 14922(CTES); Dpto. Itatí, Ramada Paso, Krapovickas et. al. 20931 (CTES); Dpto. San Luis del Palmar, rte. prov. N 3, entre ayo. Riachuelo y San Luis del Palmar, Krapovickas et al. 15520 (CTES); Dpto. Empedrado, Empedrado, Krapovickas et al. 17858 (CTES); Dpto. Mburucuyá, E Santa María, T. M.
Pedersen 4819 (BR, CTES, NY); Dpto. Saladas, E de Saladas, cerca de la rta. de Mburucuyá a Concepción, T. M. Pedersen 3689 (BR, CTES, NY); Dpto. Mercedes, cercanías de Mercedes, Ibarrola 2669 (LIL); E Guayaibí, 22 km SE de Mercedes, nacimientos ayo. Guayaibí, Tressens et. al. 2399 (CTES); Rte. 119, $28 \mathrm{~km} \mathrm{~S}$ del acceso 
a Mercedes, Tressens et. al. 2198 (CTES); Chacra Pipet, Millan $372 b$ (SI); Dpto. Goya, Colonia Carolina, Vanni 126 (CTES); Dpto. Paso de Los Libres, Cabred, Ibarrola 2231 (LIL); PARAGUAY. Cordillera: San Bernardino, Krapovickas et al. 12612 (CTES). Paraguarí: Cerro Peron, pies de Paraguarí, B. Balansa 1502 (P); Frente a Florida, orilla río Tebicuary, Krapovickas et al. 12383 (CTES); San Salvador (Alto Paraguay), Rojas 2883 (SI).

Obs. Burkart (1939) reported that it could possibly occur in Tucumán, but he had doubts because the herbarium specimen that he examined lacked fruits. As a matter of fact, S. macrosoma occurs in the northeastern provinces.

13. Stylosanthes maracajuensis Sousa Costa \& Van den Berg, Kew Bull. 58 (3): 743, fig. 1. 2003. TYPE: Brazil. Mato Grosso do Sul: Serra do Maracaju, $10 \mathrm{~km} \mathrm{~L}$. Piraputanga (Mun. Aquidaguana). Hatschbach, Schinini, J. M. Silva 58987 (holotype, MBM, not seen, isotype, CTES!, $\mathrm{K}$ !).

Perennial, suberect, branches with indument of hairs incurved, variable in density, and bristles. Stipules united, $3.5 \mathrm{~mm}$,ending in 2 appendices each with britle up to $2.5 \mathrm{~mm}$. Leaves trifoliate. Petioles 5-7 mm, slightly winged. Leaflets 7-14 $\times 1-2 \mathrm{~mm}$, linear to narrowly elliptic; inferior side with marked venation and dispersed bristles, superior side glabrous, bristles along entire margin. Inflorescences in spikes elongated up to 12 flowers. Flowers $5 \mathrm{~mm}$. Hypanthium $4 \mathrm{~mm}$. Calyx $2 \mathrm{~mm}$. Inferior tooth largest. Standard $5 \mathrm{~mm}$, rounded. Fruit with 2 segments, inferior reduced, $1 \mathrm{~mm}$; superior $2.5 \mathrm{~mm}$, with whitish hairs, and beak curved $1 \mathrm{~mm}$.

Iconography. Sousa Costa et al. (2003): Fig. 1.

Distribution and habitat. It lives in Bolivia and Brazil, in natural pastures, savanna-like habitats, and cerrado.

Additional specimens examined. BOLIVIA. La Paz: Prov. Larecaja, Vic. Amanea Charaguaya, Mandon 6 long.98 (P). BRAZIL. Mato Grosso: 23 K m de Aquidauana, 3 jul 1977, Krapovickas \& Schinini 32867 (CTES).
Obs. This species was already reported in Brazil (Sousa Costa et al., 2003) and new material collected in Bolivia extends its geographic distribution.

14. Stylosanthes montevidensis Vogel, Linnaea 12: 67. 1838. TYPE: Brazil. Hbt. in Brasilia merid.; in Montevideo, Sellow leg. (holotype B, photo 2246 B!, isotype, K!). Fig. 3.

Stylosanthes montevidensis Vogel var. intermedia Vogel, Linnaea 12: 67. 1838. TYPE: Brazil. Hbt. in Brasilia merid; in Montevideo, Sellow leg. (holotype, BUH!).

Stylosanthes juncea Micheli, Mem. Soc. Phys. Genève 28 (7): 19. 1883. TYPE: Paraguay. Itape, cuarporturs, 1874, Balansa 1507 (holotype, P!).

Stylosanthes juncea Micheli var. setosa Chodat \& Hassler, Bull. Herb. Boissier, sér. 2, 4 (9): 884.1904.

Stylosanthes montevidensis Vogel f. setosa (Chodat \& Hassler) Hassler, Repert Spec. Nov. Reg. Veg. 16: 223. 1919. TYPE: Paraguay. In regione fluminis Carimbatay, Dec.1900. Hassler 5825 (holotype, G!).

Stylosanthes montevidensis Vogel var. juncea (Micheli) Hassler f. setosa (Chodat \&

Hassler) Hassler, Repert. Spec. Nov. Reg. Veg. 16: 223. 1919. TYPE: Paraguay. Bellavista, in regione cursus superioris fluminis Apa, Dec. 1901/2, Hassler 8122. syn. nov.

Stylosanthes montevidensis Vogel f. glabrata Hassler, Repert. Spec. Nov. Reg. Veg. 16: 223. 1919. TYPE: Paraguay. Cordillera de Altos, Jan, Hassler 1798. (lectotype designated here, G!). syn. nov.

Stylosanthes montevidensis Vogel var. heterophylla Burkart, Darwiniana 3: 260. 1939. TYPE: Brazil. Paraná: Capao Grande, Ponta Grossa, in campo, 17 Feb.1911, Dusen s. $n$. (holotype, SI 1479!). syn. nov.

Perennial, branches erect, with few leaves, appearing juncoid, indument with white, incurved, weak hairs variable in density; bristles $4.5 \mathrm{~mm}$ at base of stipule insertion. Leaves trifoliate. Stipules glabrous, with bristles, veins marked, united part 9 $\mathrm{mm}$, free part $4.5 \mathrm{~mm}$, subtriangular, acute, weak hairs and bristles along margins and at tip of free part. Petioles $4.5-7 \mathrm{~mm}$, with white bristles and hairs. Petioles short, with tuft of white hairs at base of each leaflet. Leaflets $9-23 \times 1-2 \mathrm{~mm}$, terminal largest, inferior side with marked venation and hairs 
applied, superior side with some white weak hairs and gland dots, margin with rigid bristles reddish at base. Spikes dense globose, contracted, with 3 ramifications at base, up to 30 flowers. Flowers, 7 $\mathrm{mm}$, with 2 basal bracteoles. Hypanthium $3 \mathrm{~mm}$. Calyx $2.5 \mathrm{~mm}$, campanulate, 5 teeth, 4united, superior 1 free and shortest. Petals unguiculate; Standard rounded, $6.5 \mathrm{~mm}$. Wings oblong. Keel slightly incurved. Fruit with only 1 segment, fertile, $5 \times 2.5 \mathrm{~mm}$, with conspicuous veins, hairs yellowish, dense in concavity of beak. Beak spiral.

Iconography. Burkart (1939): fig. 17.

Distribution and habitat. Stylosanthes montevidensis is found in Argentina, Bolivia, south eastern and eastern Brazil, Paraguay, and Uruguay, in fields, sandy soil, and dunes with rocky outcrops at (416) 900-1600 $\mathrm{m}$ a.s.l.

Additional specimens examined. ARGENTINA. Buenos Aires: Lincoln, Schultz 5708 (CTES). Catamarca: Dpto. El Alto, Tintigasta, B. Ahumada 12 (LIL). Chaco: Dpto. Bermejo, Campo Vélaz, Schultz 9950 (CTES); Dpto. 1 de Mayo, Clnia. Benítez, Schultz. 14322 (CTES); Dpto. 12 de Octubre, Hermoso Campo, Schultz 8945 (CTES); Dpto. San Fernando, Basail, Schultz 13086 (CTES). Córdoba: Dpto. Punilla, Copina, D. Grassi 2281 (LIL); Dpto. Calamuchita, Cumbrecita, Krapovickas 7597 (LIL); Dpto. Colón, E de Ayala, Alessandria 6950 (CTES);Dpto. San Alberto, Mina Clavero, T. Meyer 13382 (LIL); Dpto. Cruz Alta, km 754, F.C.N., Venturi 1519 (SI); Las Cejas, Venturi 2117 (SI); Valle de los Reartes, Castellanos 487 (SI). Corrientes: Dpto. Ituzaingó, $25 \mathrm{~km}$ al E de Ituzaingó, Rte. 12, Krapovickas et al. 12008 (CTES); Dpto. Bella Vista, Paraje Muchas Islas, Carnevali 2385 (CTES); Dpto. Santo Tomé, Cuay Grande, Ibarrola 1608 (NY); Dpto. Concepción T. M. Pedersen 4475 (NY); Dpto. Gral. Paz. 1 Sección, Angostura, T. M. Pedersen 4455 (NY); Dpto. Saladas, Pago Alegre, T. M. Pedersen 9056 (NY); Dpto. San Martín, Yapeyú, Ibarrola 1893 (NY); Dpto. San Roque, M.F. Mantilla, Ibarrola 2882 (NY); Dpto. Monte Caseros, Monte Caseros, Schulz 18898 (CTES). Entre Ríos: Dpto. Federación, Ayo. La Virgen, Troncoso et al. 2471 (SI); Dpto. Concordia, Ayo. Gualeguaycito, Burkart 1023 a (SI); Dpto. Feliciano, San José de Feliciano, E La Emilia, Nicora et al. 7730 (SI). Formosa: Dpto. Pilagá, rte. 81 al km 101, Morel 7503 (LIL); Dpto. Pilcomayo, ruta $86 \mathrm{~km}$ 52, Morel 5214 (LIL). Dpto. El Colorado, Pto. Vélaz, E El Tano, Villa Escolar, J. G Fernández 555 (CTES). Jujuy: Dpto. Capital, Quebrada de Chañi, Schreiter 11083 (LIL). La Pampa: Quintero 18 (LIL); La Pampa. Realicó. Schulz 5442 (LIL). Misiones: Dpto. San Pedro, Fracrán a San Pedro, ruta. 14, Schwindt 3339 (CTES); Dpto. Candelaria, Loreto, Krapovickas et al. 20768 (CTES); Apóstoles, E. Closs 1911(B). Salta: Dpto. Rosario de la Frontera, Rosario de la Frontera, O'Donell 4739 (LIL). Santa Fe: Reconquista, F. C.S.F., Meyer 607 (SI). Santiago del Estero: Ojo de Agua, W. Gregory et al. 9524 (LIL). Tucumán: Dpto. Tafí, Siambón, Olea 86 (CTES); Dpto. Burruyacú, Sierra de Medina, Venturi 2616 (SI). BOLIVIA. s.1., Bang 2150 (NY). BRAZIL. Mina Gerais: Pirapora, Krapovickas et al. 42880 (CTES); Mun. Pirapora, Rd. Pirapora-Corinto, km 5-10 Hatschbach et al. 64130 (C). Paraná: Serrinha, en campo, Dusén13718 (NY, SI); Vila Velha, alrededores del camping, Krapovickas et al. 40771 (CTES). Rio Grande do Sul: 14 km de Sâo Francisco de Paula, camino a Canela, Krapovickas et al. 44694 (CTES); Granja Geres, Mun. Tupanciretan, prop. Joia, Pereira 8618 (B); Sto. Angelo, p. Cruz Alta, Rambo 53025 (B); Vila Oliva, p. Caxias, Rambo 55018 (B); Est. De silvicultura, Sta. María, Camargo 131 (B). Santa Catarina: Lajes, Morro Pinheiro Seco, $3 \mathrm{~km}$ E of Lajes, Smith \& Reitz 10035 (NY). São Paulo: Rio Claro, Lofgren 572 (C). PARAGUAY. "Paraguay, in campis, Cordillera de Altos, Jan.”, Hassler 3781 (G!) sintype of Stylosanthes montevidensis Vogel var. juncea (Micheli) Hassl. f. setosa Hassl. "Pr. Paraguary, Dec. 1900”, Hassler 6573 (G!) sintype of Stylosanthes montevidensis Vogel f. glabrata Hassl. Caaguazú: $5 \mathrm{~km}$ E de Caaguazú, ruta. 2, Krapovickas et al. 12494 (CTES). Central: Morong 399 (NY); Campo, E Primavera, Jorgensen 4817 (NY). Misiones: E La Soledad, $3 \mathrm{~km} \mathrm{~S} \mathrm{de}$ Santiago, $56^{\circ} 46^{\prime} \mathrm{W}, 27^{\circ} 10^{\prime} \mathrm{S}$, Schinini y Vanni 26102 (CTES). Presidente Hayes: Ruta Transchaco, km 58, desvío a Loma Porá, Krapovickas \& Cristóbal 43214 (CTES). URUGUAY. Canelones: Prop. Montevideo, Gibert 1002 (K). Cerro Largo: río Negro, E Palleros, Rosengurtt B-79 (NY). Salto: Near Termas Arapay, T. M. Pedersen 16183 (C). 
Obs. It is the species with thw southest distribution range of the genus, occuring in sympatry with $S$. hippocampoides and $S$. rostrata.

15. Stylosanthes nervosa J. F. Macbr., Publ. Field Mus. Nat. Hist., Bot. Ser. 13, part 3: 411. 1943. TYPE: Peru. Cajamarca, near confluence of rivers Chinchipe and Marañón, Weberbauer 6215 (holotype, NY, not seen, isotype, US, photo 0001967 US !). Figs. 3, 9.

Perennial, erect, up to $25-30 \mathrm{~cm}$ high, branches ascendent to erect, with indument of dense hairs incurved and rigid bristles. Stipules united, with 2 free extremities, on both sides of petiole, triangular, 3.5-4 mm, ending in 1 seta. Leaves trifoliate. Leaflets 7-12 × 1.5-3 mm, narrow-elliptic, inferior side with indument dense of incurved hairs, hyaline, bristles on middle vein and lamina, leaflet margins dentate, with 1 seta at base of each tooth; superior side with same indument as inferior side, lamina cleft around middle vein, rendering leaflets carinate. Inflorescences in dense spikes, 1- to 7-flowered. Bracts inflorescence similar to leaflets, terminal largest, floral bracts with 1 leaflet, 2 hyaline bracteoles, external largest and covering internal, which is small and ciliated. Flowers $6.5 \mathrm{~mm}$ yellow-clear. Hypanthium $7 \mathrm{~mm}$. Calyx $2.5 \mathrm{~mm}$. Petals unguiculate. Standard obovate. Wings oblong. Keels lightly incurved. Fruit with 2 segments, $11.5 \mathrm{~mm}$, inferior segment $4 \mathrm{~mm}$, with yellow hairs dense on veins, superior segment 7 $\mathrm{mm}$, with similar indument to inferior segment. Beak $4 \mathrm{~mm}$, with 1 curvature closed at apex, completely yellow when dry.

Distribution and habitat. Stylosanthes nervosa was collected in Venezuela, Bolivia (Mohlenbrock, 1957), Peru, Paraguay, and Argentina, in grasslands with rocks and rocky outcrops.

Additional specimens examined. ARGENTINA. Corrientes: Dpto. San Martín, Pedregal Guaviraví, 15 Feb. 1979, Perego s.n. (CTES); Dpto. Paso de los Libres, Laguna Mansa, Krapovickas et al. 21705 (CTES); Paso de los Libres, Huidobro 3699 (LIL); Tapebicua, Ibarrola 2006 (LIL); Dpto. Monte Caseros, Monte Caseros, Ibarrola 2310 (BAB); $8 \mathrm{~km} \mathrm{~N}$ de Monte Caseros, prox. Ayo. Ceibo, Vanni et al 4656 (CTES). PARAGUAY.
Concepción: $1 \mathrm{~km}$ E de Huguá Poi, Dematteis et al. 3222 (CTES). PERU. Cajamarca: Celedin, Rio Marañón, opposite Balsar and up stream $1 \mathrm{~km}, P$. Hutchinson \& J.K. Wright 5437(M).

Obs. This species was previously considered closely related to $S$. scabra Vogel, but specimen comparisons showed that they are indeed different species. I studied a duplicate of the specimen Ibarrola 2310 (BAB), cited by Mohlenbrock (1957). The locality of this specimen was doubtful, and this was the only Argentinean material from Corrientes that the author saw, confirming its occurrence in the Province of Corrientes. A recent collection confirms the species' presence in Corrientes after 60 years. This species is not represented in the world's herbaria and its distribution is somewhat unusual. In Argentina, it also occurs in the province of Salta. It is here reported in Paraguay for the first time.

16. Stylosanthes recta Vanni, Candollea 50 (1): 247. 1995. TYPE: Paraguay. Boquerón: Parque Valle Natural, 25 km S de Filadelfia, 7 Dec.1992, Krapovickas \& C. L. Cristóbal 44219 (holotype, CTES!, isotype, G!, SI!). Fig. 10.

Perennial, erect, 40-50 $\mathrm{cm}$ high, densely branched; branches with bristles up to $4 \mathrm{~mm}$ and whitish curved hairs of variable density. Leaves trifoliate. Stipules 9-16 mm, united at base, where they are papiraceous, translucid, with dense hairs, bristles and weak hairs, free at apex, ending in 1 seta. Leaflets 9-26 × 3-9 mm, elliptic, narrow, mucronate, superior and inferior sides with weak hairs, variable in density and bristles on veins. Inflorescences in spikes globose, dense, up to 9 flowers. Bracts similar to leaflets, with dense, short hairs and other weak hairs. Bracteoles 2, hyaline, protecting each flower. Flowers yelloworangish, $9 \mathrm{~mm}$. Hypanthium $6 \mathrm{~mm}$. Calyx $3 \mathrm{~mm}$, campanulate, 5 teeth, 2 superior teeth united, the rest free, 4 obtuse, inferior acute and longest. Petals unguiculate. Standard rounded, 4-6 $\mathrm{mm}$. Wings oblong. Fruit with 1 segment, $6-8 \times 2.5-4 \mathrm{~mm}$, slightly reticulate, pubescent, hairs dense on beak. Beak straight, with slight apical curvature when immature.

Distribution and habitat. It lives in northwestern Paraguay, in savanna-like habitats with trees and 
Bol. Soc. Argent. Bot. 52 (3) 2017
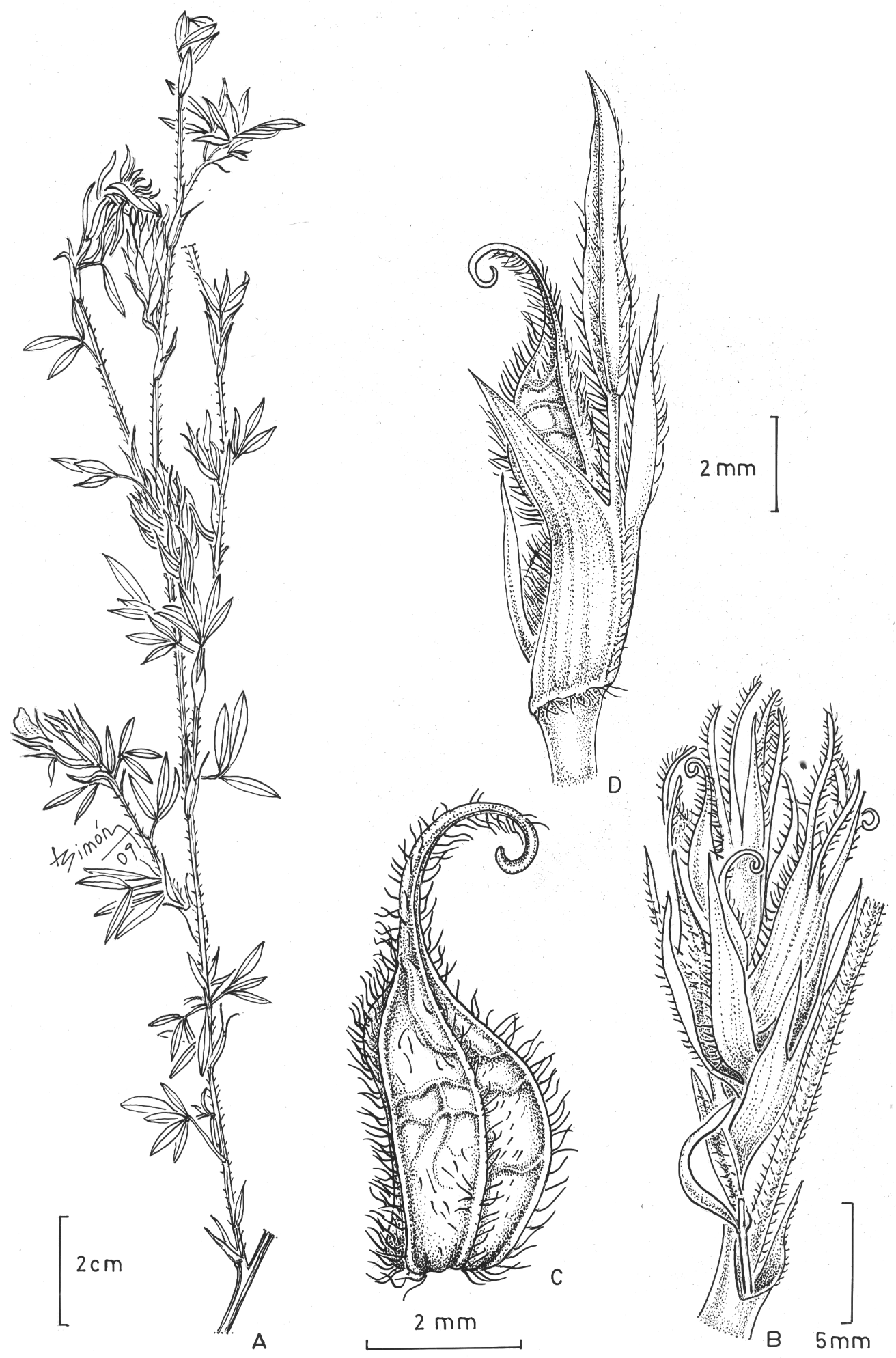

Fig. 9. Stylosanthes nervosa. A Plant. B. Inflorescence. C. Superior segment. D. Fruit. (Vanni 4656). 


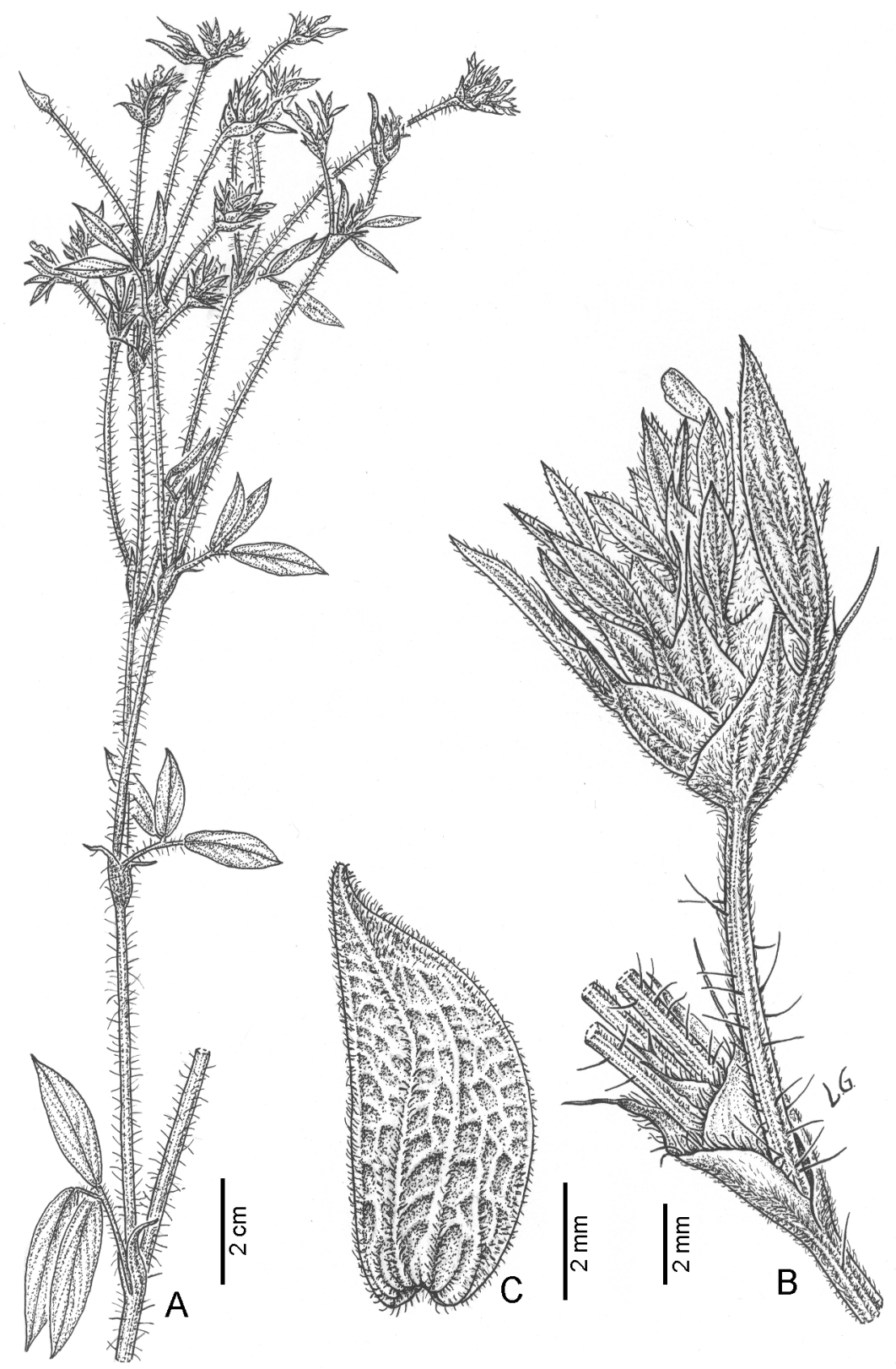

Fig. 10. Stylosanthes recta. A. Plant. B. Inflorescence. C. Fruit. (Vanni 2173).

"espartillares" (vegetation dominated by Spartina argentinensis Parodi).

Additional specimens examined. PARAGUAY. Boqueron: Estancia San Ramón, 2241'33"S, $60^{\circ} 30^{\prime} \mathrm{W}^{\prime}$, L. Perez \& al. 2531 (CTES); "Parque Valle Natural, Vanni et al. 2448 (CTES, G, LIL, NY); 9 km de Loma Plata, prop. de Ebenfeld, Vanni et al. 2173 (CTES); Est. Exp. Neuland, Vanni et al. 2495 (CTES). Nueva Asunción: Gral. Eugenio A. Garay, picada al S de la delegación de Gobierno de Nueva Asunción, Charpin \& Ramella AC 21545 (G). Pte. Hayes: Isla Poí, 24 km N de ruta TransChaco, km 415, Krapovickas et al. 44356 (CTES); Estancia Yrenda, $22^{\circ} 48^{\prime} \mathrm{S}, 5^{\circ} 33^{\prime} \mathrm{W}^{\prime \prime}$, L. Perez et al. 2995 (CTES). 
Obs. From the six species mentioned to occur in Paraguay (Blake, 1920; Burkart, 1939; Mohlenbrock, 1957), S. recta is similar to $S$. macrosoma, differing only in fruit morphology. In both species, the fruit possesses only one segment, but in the latter species, the segment is $8-9 \times 3 \mathrm{~mm}$ and is covered by reddish hirsute hairs up to 0.5 $\mathrm{mm}$ in the middle line of the spiral beak, whereas in the new taxon, the segment is $6-8 \times 2.5-4 \mathrm{~mm}$ and is covered by whitish weak hairs especially in the superior half of the segment, and the beak is straight.

17. Stylosanthes rostrata (Burkart) Vanni, Novon 19 (2): 259-260. 2009.

Basionym: Stylosanthes gracilis Kunth var. rostrata Burkart, Darwiniana 3 (2): 251, fig. 16 r-s. 1939. TYPE: Argentina. Corrientes: Granja de Bella Vista, 18 July1905, C. Spegazzini y Girola 54, Min. Agr. 14648 (holotype SI!).

Perennial, branches prostrate to suberect, with bristles and short, white, incurved hairs. Leaves trifoliate. Stipules $11 \mathrm{~mm}$, united to one another and to petiole base, free in superior half where they are point-tipped, with bristles and some incurved hairs. Petioles 5-6 mm. Leaflets 12-15 × 2-3 mm, narrow-elliptic, mucronate, with 1 bristle at apex, lateral asymmetric, terminal largest; inferior side with some weak hairs, veins conspicuous and white, bristles dense on top of venation, margin dentate with 1 seta on each tooth, superior side with isolated bristles. Inflorescences terminal or subterminal, spikes dense, up to 8 flowers. Bracts similar to stipules, with central lamina ca. $8 \mathrm{~mm}$. 2 hyalinebracteolesat base of each flower. Flowers $6 \mathrm{~mm}$. Fruitwith only 1 segment, inferior rarely developed, $6 \times 3 \mathrm{~mm}$, conspicuous longitudinal veins, glabrous, few thick hairs near beak. Beak open, $1.5 \mathrm{~mm}$, and slightly curved only at apex.

Iconography. Vanni (2009): Fig. 1.

Distribution and habitat. This species grows in northeastern Argentina (Corrientes) and Uruguay, in savanna-like habitats.

Additional specimens examined: ARGENTINA. Corrientes: San Roque, ruta 12 y entrada a Est. Mantilla, Vanni et al. 4657 (CTES). URUGUAY.
Colonia: Riachuelo, Herter 85562 (NY); La Colonia, (río de la Plata), Feb.1918, L. Hauman s.n., (BR).

Obs. It is closely related to $S$. hippocampoides, from which it can be distinguished by the fruit, especially the shape of the beak that forms a closed spiral in S. hippocampoides. Mohlenbrock (1957) considered the name $S$. gracilis var. rostrata Burkart as a synonym of $S$. montevidensis without studying the type material of Burkart's variety. Stylosanthes montevidensis can be distinguished from $S$. rostrata by its juncoid habit with few leaves and its fruit with a spiral beak and yellowish hairs. Having collected it again in 2011, after more than 100 years in which it was not found, I confirm that it is a valid species and not only a variation of other sympatric species.

18. Stylosanthes ruellioides Martius ex Benth., Fl. Bras. 15 (1A): 90. 1859. TYPE: Brazil. Hbt. in Minarum campis editis ad Serra Caraca et Serra Itambé. Martius (holotype, M, not seen, photo 6268 M!). Fig. 11.

Stylosanthes tomentosa M. B. Ferreira \& N. M. Sousa Costa, Soc. Bot. Brasil Anais 28: 102. 1977. TYPE: Brazil. Minas Gerais: Datas, 20 km após Datas-Serro, $800 \mathrm{~m}, 1^{\circ} 37^{\prime} \mathrm{S}, 4^{\circ} 37^{\prime} \mathrm{W}$, Jan. 191977, N. M. Sousa Costa 794 (holotype, RB!). syn. nov.

Perennial, shrubs, up to $1.5 \mathrm{~m}$ high, densely branched. Branches with indument of dense, whitish, and some what appressed hairs, bristles dense, thickat base, abundant throughout plant. Leaves trifoliate. Stipules 6-13 $\times 2 \mathrm{~mm}$, with indumentof branches, united to one another and at leaf base, ending in 1 arista. Leaflets 15$45 \times 3-10 \mathrm{~mm}$, narrow-elliptic, acute, inferior side with conspicuous white veins, hairs whitish, dense, and bristles with reddish, thickened base; superior side with similar indument as inferior side. Inflorescences, spikes dense, 1 to 5 -flowered. Bracts highly, similar to stipules. Flowers $15 \mathrm{~mm}$, yellow and red veins in standard. Hypanthium 5 $\mathrm{mm}$. Calyx $6 \mathrm{~mm}, 5$ teeth, inferior largest. Standard $14 \mathrm{~mm} \times 8 \mathrm{~mm}$, rounded, cuneate at base, slightly emarginated. Wings $14 \times 4 \mathrm{~mm}$, unguiculate, claws $4 \mathrm{~mm}$. Keel incurved, $12 \times 2 \mathrm{~mm}$. Fruit with 1 (2) segments, inferior generally aborted, superior 4 


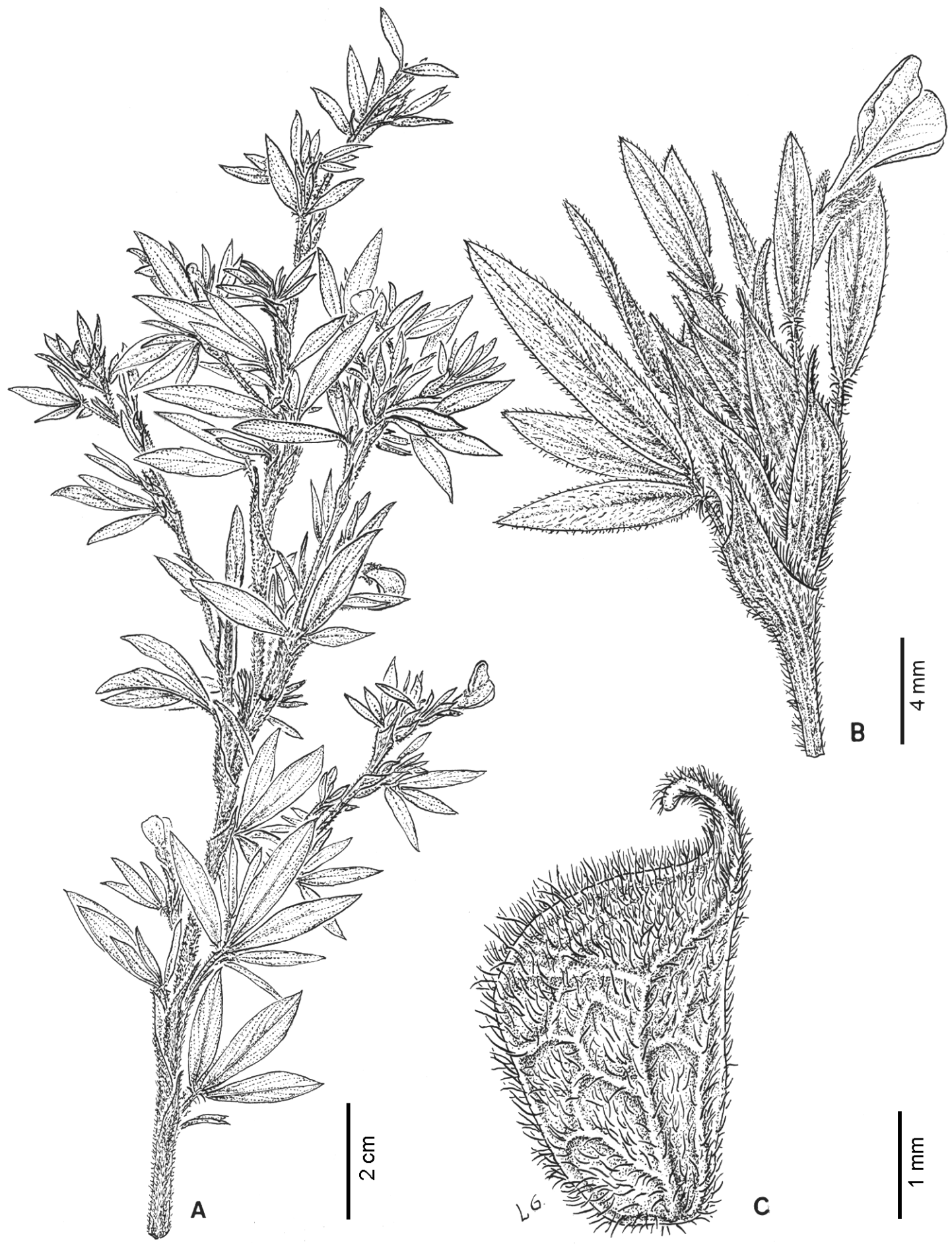

Fig. 11. Stylosanthes ruellioides. A. Plant. B. Inflorescence. C. Fruit. (Arbo et al. 5189). 
$\mathrm{mm}$, with dense, castaneous hairs. Beak $1.5 \mathrm{~mm}$, curved, pubescent.

Distribution and habitat. It was collected in southeastern Brazil, on mountain slopes at 900$1300 \mathrm{~m}$ a.s.l.

Additional specimens examined. BRAZIL. Minas Gerais: Minas Gerais, A. St. Hilaire 864 (P); Grao Mogol est. rio Ventania, I. B. Cavalcanti et al. SPF $42893(\mathrm{~K})$; Mun. Jaboticatuba, Serra do Cipó, Fda. Palacio, Hatschbach et al. 31599 (CTES); Serra do Cipó, $25 \mathrm{~km}$ ao N, Hatshbach et al. 28837 (C, CTES, NY); Serra do Cipó, Drade 14861 (RB); Cerra do Cipó, km 137, Duarte 2602 (RB); Mun. de Diamantina, Guinda, Hatschbach et al. 44662 (CTES); Alvorada de Minas, alrededores de Itapanhoacanga, base de la serra do Espinhaco $18^{\circ} 46^{\prime} \mathrm{S}, 43^{\circ} 27^{\prime} \mathrm{W}$, Arbo et al. 4306 (CTES); Diamantina, bairro da Cazuza, cachoeira da Toca, $18^{\circ} 14^{\prime} \mathrm{S}, 43^{\circ} 36^{\prime} \mathrm{W}$, Arbo et al. 5189 (CTES); Diamantina, Pirusuca, Pereira 1599 (RB); Diamantina, and humber, Glaziou 19033(C, P); Grao Mogol, arrededores Minas Gerais, Hatschbach 41233 (C).

Obs. The distribution of this rare species is particularly striking, as it is localized to the mountains of Minas Gerais.

19. Stylosanthes scabra Vogel, Linnaea 12: 6970. 1838. TYPE: Brazil. Hbt. in Brasil merid.: in Serra da Maeda s. d. F. Sellow s.n. (holotype BHU, photo2248 F!). Fig. 3.

Stylosanthes seabrana B. L. Maass \&'t Mannetje, Novon 12 (4): 497-499. 2002. TYPE: Brazil. Bahia: State Rd. BA 052, from Irecê $80 \mathrm{~km}$ to Xique-Xique, $10^{\circ} 56^{\prime} \mathrm{S}, 42^{\circ} 29^{\prime} \mathrm{W}, 29$ June 1983 , L. Coradin, R. Baker, F.B. de Souza, R. M. Harley \& S. Linintong LC 6261 (holotype, CEN, not seen, isotype, $\mathrm{K}$ !, $\mathrm{M}$ !).

Perennial, erect, $50 \mathrm{~cm}$ high, ramified, branches with indument of weak, white hairs of variable density and bristles up to $2 \mathrm{~mm}$. Leaves trifoliate. Stipules united at leaf base, free at apex, where they are point-tipped, with bristles. Petioles 4-6 $\mathrm{mm}$, with whitish hairs. Leaflets $8.5-15 \times 2-5 \mathrm{~mm}$, elliptic, superior side glabrous, inferior side with gland dots, with marked veins, white or green, clearer than the rest of lamina, with bristles on veins and margins. Inflorescences in dense spike, always developed axillary, up to 5 flowers, every flower protected by 1 bract, similar to leaves, but smaller, 2 bracteoles for each flower and 1 feathery, extrafloral stipe. Flowers $5.5 \mathrm{~mm}$, hypanthium 5 $\mathrm{mm}$. Calyx campanulate, $2 \mathrm{~mm}$, tube $1 \mathrm{~mm}, 5$ teeth, 2 superior united and rounded, inferior largest and ciliate, $1 \mathrm{~mm}$. Standard $5 \mathrm{~mm}$, rounded, emarginate. Wings oblong. Keel slightly incurved. Fruit with 2 segments, $11 \times 2.5 \mathrm{~mm}$, with dense, weak, and white hairs, venations marked, superior segment with apically incurved beak $2 \mathrm{~mm}$.

\section{Iconography. Burkart (1939): fig. 14 a, g.}

Distribution and habitat. Stylosanthes scabra inhabits in Colombia, Venezuela, Ecuador, Peru, Bolivia, Paraguay, Argentina, and northeastern, central-western, southeastern and southern Brazil, in fields, mountain slopes, and lomadas at 420-1900 $\mathrm{m}$ a.s.l. It is cultivated in Paraguay, Colombia and Australia.

Additional specimens examined. ARGENTINA. Jujuy: Dpto. Ledesma: Ledesma, Spegazzini (Min. Agr. 13943) (SI). Salta: Dpto. Capital, lomadas 1 ayo. ruta Salta a San Lorenzo, 21 Mar. 1979, J. Fernández s.n. (CTES); $3 \mathrm{~km}$ de Salta, camino a San Lorenzo, La Loma, Krapovickas et al. 35971 (CTES); Lomas del Mecidor, Feb. 1925, Inostroza s.n. (LIL). BOLIVIA. Chuquisaca: Tomina, Los Naranjos, Saravia Toledo 11114 (CTES). Santa Cruz: Gutierrez, orillas del río Piray, $8 \mathrm{~km} \mathrm{E} \mathrm{de}$ Portachuelo, Krapovickas et al. 31561 (CTES); Chiquitos, $2 \mathrm{~km}$ W de San José, km 263, del ferrocarril, $60^{\circ}, 47^{\prime} \mathrm{W}, 17^{\circ} 47^{\prime} \mathrm{S}$, Krapovickas et al. 36531 (CTES); Nuflo de Chávez, 3 km S de Ascención de Guarayos, $63^{\circ} 6^{\prime} \mathrm{W}, 15^{\circ} 43^{\prime} \mathrm{S}$. Ayo. San Joaquín, Krapovickas et al. 31801 (CTES); Andrés Ibañez, ciudad de Sta. Cruz, Coimbra 548 (NY); Tarija: Santa Cruz, rte. Tarija Villa Montes, Entre Ríos, Krapovickas et al. 19087 (CTES). BRAZIL. Amazonas: Glaziou 9733 (C). Bahia: Bahia, in orilloris aridi, 1830, Salzman s.n. (P); Lagoa da Eugenia, near Camaleao, Harley et al 16248 (P); Jacobina, Krapovickas 12823 (CTES); Mun. de Lancois, Rio Lancois, Hatschbach et al. 56954 (CTES); Jaguaquara, estrada BR. 4, Krapovickas 12777 (CTES); Chapada occidental 
da Bahia, islets and branches of rio Corriente, by Correntina, $44^{\circ} 38^{\prime} \mathrm{W}, 13^{\circ} 20^{\prime} \mathrm{S}$, Harley 21622 (NY). Distr. Federal: Brasil inter, Martius 1124 (P); Brasilia. Warming 3168 (C); BR. 20, entrada a Formosa, Arbo et al. 3441 (CTES); Estrada entre Taguatinga-Brazlandia, $15^{\circ} 46^{\prime} \mathrm{S}, 48^{\circ} 4^{\prime} \mathrm{W}$, Kirkcbride 1640 (CTES). Rio de Janeiro: Rio de Janeiro, Glaziou 1375 (BR). Minas Gerais: Minas Gerais, Chapada Praevium, Martius 757 (M); Clausen 905(P); Gouveia, rib. do Tigre, Hatschbach 56954 (NY); Prope Caza Blanca, Prov. De Minas Gerais, Riedel 735 (P). Mato Grosso do Sul: Alrededores de Campo Grande, camino a Sidrolandia, Krapovickas et al. 34531 (CTES). Sao Paulo: Mun Campinas, beira rd. D. Pedro, $1 \mathrm{~km}$ ao trevo p/ Magi-Mirin, H. Freitas Leitao Filho 1787 (NY); Campos das sete Lagoas, $3.7 \mathrm{~km} \mathrm{NNW}$ of padua Sales, $22^{\circ} 18^{\prime} \mathrm{S}, 47^{\circ} 10^{\prime} \mathrm{W}$, cerrado, Eiten et al. 2742 (NY). ECUADOR. Loja: $5 \mathrm{~km} \mathrm{NE}$ of La Loma, J.E. Madsen et al. 7789 (K). PARAGUAY. Alto Paraguay: Pto. Casado, Arenas 729 (CTES). Amambay: $22^{\circ} 45^{\prime} \mathrm{S}, 56^{\circ} 15^{\prime} \mathrm{W}$, rutas 3 y 5 , Schinini et al. 20524 (CTES); Cerro Corá, Schinini et al. 20284 (CTES); ruta. 3, $35 \mathrm{~km}$ SE de Bella Vista, Schinini et al. 20568 (CTES). Canindeyú: Reserva biológica Mbaracayú, Caballero Mármori 2091 (CTES). Chaco: Cerro León, lomada al E de la meseta central, Charpin. \& Ramella A C 21661(CTES); Cap. Pablo Lagerenza, $20^{\circ} 20^{\prime} \mathrm{S} 60^{\circ} 20^{\prime} \mathrm{W}$, Ramella 2170 (CTES). Pte. Hayes: Est. Exp. Chaco Central M.A.G. G.T.Z. Col de leguminosas introducidas CSIRO-110361, indentif. S. aff scabra, Vanni \& Lopez 3988(CTES); Boquerón, Filadelfia, Isla Poí. "Unica”, componente 2 de la mezcla, 27 Jan. 2006, Glatzle s.n. (CTES). PERU. Tumbes: Tumbes, La Cruz, Sagástegui 14615 (NY). VENEZUELA. In savanna Nagrea, Warming 101 (C).

Obs. This species is here reported in Paraguay for the first time.

20. Stylosanthes sericeiceps S. F. Blake, Contr. $U$. S. Natl. Herb. 20 (13): 524. 1924. TYPE: Venezuela. Mérida: Lagunillas, 1000 m, 6 Oct. 1921, Jahn 678 (holotype, US, photo 0001957 US!).

Perennial, with ascending branches, covered by soft, whitish hairs. Leaves trifoliate. Stipules united, forming a tube, point-tipped. Petioles $3 \mathrm{~mm}$. Leaflets $10 \times 2 \mathrm{~mm}$, elliptic. Spikes dense terminal.
Flowers $7 \mathrm{~mm}$. Fruit with 1 or 2 fertile segments, superior $5 \mathrm{~mm}$, with a curved, hook-like beak.

Distribution and habitat. It is found in Venezuela, in savanna-like habitats.

Additional specimens examined. VENEZUELA. $36 \mathrm{~km}$ SW de Mérida, along rute S. Cristóbal, F. J. Breteler 4070(GENT, US); Chama valley, $22 \mathrm{~km} \mathrm{SW}$ of Mérida, F. J. Breteler 4546 (GENT, US).

21. Stylosanthes sympodialis Taubert, Verh. Bot. Vereins Prov. Brandenburg 32 (1): 19-20. 1890. TYPE: Ecuador. pr. Chanduy, Spruce 6373 (holotype, B, photo $2249 \mathrm{~B}$ ! isotype, $\mathrm{K}$ !).

Perennial plant, branches prostrate, $50 \mathrm{~cm}$ high, indument with dense, whitish, curved hairs, and bristles. Leaves trifoliate. Stipules 7-10 mm, united to one another and at base of petiole, with whitish hairs and dense bristles, free in upper third, where they are point-tipped and ending in 1 bristle. Leaflets $10-20 \times 2-5 \mathrm{~mm}$, narrow-elliptic, pale green, inferior side with weak hairs and bristles on veins and margin, superior side with weak delicate hairs, without bristles. Spikes dense, terminal, up to 6 flowers. Bracts $5 \mathrm{~mm}$, hyaline at base, with dense reddish hairs and bristles, similar to trifoliate leaf or simply ending in 1 leaflet. Flowers $7 \mathrm{~mm}$. Fruits with 2 segments, inferior 2 $\mathrm{mm}$, pubescent, superior $5 \mathrm{~mm}$, with long, dense, yellow hairs. Beak $2 \mathrm{~mm}$, curved, closed.

Distribution and habitat. This species inhabits in Ecuador, including Galápagos Islands, and Peru, in savanna-like habitats.

Additional specimens examined. ECUADOR. Guayas: Punta Centinela, $2^{\circ} 11^{\prime} \mathrm{S}, 80^{\circ} 49^{\prime} \mathrm{W}$, Svenson 11258 (NY). Manabi: Manta, E. Asplun K 15976(B, K). Santa Elena: peninsula W of Guayaquil, Caldeabank 22 (K); El Oro, Arenillas, E. Asplund 15753 (C). Galapagos Islands: Albemarle Island, Tagus cove, Stewart 1695 (NY); Charles Island, G. Baur 92(K); Isabela, H.H.van der Wesff 1013 (K); Bahia Santa María (Floruana Charles) Sr. Cruz farm. A. \& H. Andersen 1523 (C); Isla Albany, medium part, A. \& H. Andersen 1835 (C); Volcan Alcedo, W side Bahia Urvina, H. \& O. Harman 1766. 
22. Stylosanthes tuberculata S. F. Blake, Proc. Biol. Soc. Washington 33: 48-49. 1920. TYPE: Bahamas. New Province Islands, Southwest Landing, 11 Feb. 1905, Britton 3336 (holotype US, isotype, NY!). Fig. 12.

Perennial, erect, ca. $50 \mathrm{~cm}$ high. Branches with whitish weak hairs and bristles basally highly thickened, very abundant throughout plant. Leaves trifoliate. Stipules 6-7 mm, united, free apically, where they are point-tipped. Petioles 2.5-5 mm. Leaflets $10-14 \times 3.5-4 \mathrm{~mm}$, elliptic to obovate, mucronate, inferior and superior sides with abundant bristles dense along leaflet margins. Spikes globose, up to 7 flowers. Flowers $5 \mathrm{~mm}$. Fruit with 2 pubescent segments similar to those of $S$. bracteata, superior $5 \mathrm{~mm}$, castaneous, with dense, short, whitish hairs; inferior segment 1.5 $\mathrm{mm}$. Beak glabrous, forming a well-developed open hook.

Distributionand habitat. It is found in Costa Rica, Bahamas, Cuba, Colombia, Ecuador, Venezuela, and Peru, in savanna-like habitats at 100-300 m a.s.l.

Additional specimens examined. COLOMBIA. Valle: Dagua Valley, Ellsworth Killip 5431 (NY). COSTA RICA. Guanacaste: Santa Rosa. National Park, Liesner $4820 A$ (NY). PERU. s.l. Weberbauer 6457 (US). Cajamarca: Pucará, 127 km Olmos, $P$. C. Hutchinson et al. $3554(\mathrm{~K})$.

23. Stylosanthes viscosa (L.) Sw., Prodr. (Sw.): 108. 1788.

Basionym: Hedysarum hamatum L. var. viscosum L., Syst. Nat. ed. 10, 2: 1170. TYPE: Jamaica. Loto pentaphyllo siliquoso villoso similis anonis non spinosa, foliis cisti instar glutinosis \& odoratis. In Sloane, Voy. Jamaica, 1: 186, t. 119, f. 1.1707 (lectotype designated by Kirkbride \& Kirkbride, 1987: 456.). Fig. 3.

Stylosanthes debilis M. B. Ferreira \& Sousa Costa, Empresa de Pesquisa Agropecuaria de Minas Gerais 48-49. 1979. TYPE: Brazil. Mina Gerais: Divisa Minas Gerais, canal Saô Simâo, BR 365, 40 m, $19^{\circ} 00^{\prime} \mathrm{S}, 050^{\circ} 30^{\prime \prime} \mathrm{W}$. 14 May 1976, N. M. Sousa Costa 619 (holotype, RB!). syn. nov.

Stylosanthes pilosa M. B. Ferreira \& Sousa Costa, Soc. Bot. Brasil Anais 28: 102. 1977. TYPE:
Brazil, BR 365, Buritizeiro-Pirapora, km 201, 15 Apr. 1976, M. B. Ferrerira et N. S. Costa 362 (holotype, RB!). syn. nov.

Perennial, branches suberect. Dense white hairs throughout plant and bristles, which secrete a viscous substance, rendering plant sticky. Stipules unitedat base of petiole, free in apical part where they are point-tipped. Petioles short, $5 \mathrm{~mm}$. Leaves trifoliate. Leaflets $12-19 \times 3-5 \mathrm{~mm}$, inferior side with white, highly conspicuous venation, bristles dense on margins and middle vein, and white incurved hairs; superior side with same indument as the inferior side. Inflorescences, spikes globose or elongated, up to 9 flowers. Bracts similar to stipules, 2 hyaline bracteoles at base of each flower. Flowers $4.5 \mathrm{~mm}$. Hypanthium $3 \mathrm{~mm}$. Calyx $2 \mathrm{~mm}$, inferior tooth largest. Petals unguiculate. Standar drounded, slightly emarginate.Wings oblong. Keel falcate. Fruit with only 1 segment, $4 \mathrm{~mm}$, reticulate, pubescent, hairs white, short, rigid, and glands reddish, rounded. Beak curved, with the same indument as the segment.

Distribution and habitat. It lives in the United States, México, El Salvador, Cuba, Colombia, Surinam, French Guyana, Venezuela, Bolivia, Paraguay, Argentina, and northeastern, southeastern, and southern Brazil. It occurs in diverse habitats from lomadas with sandy soil, places with rocky outcrops and fields at high altitudes on disturbed soils at (300) 617-2100 m a.s.l.

Additional specimens examined. ARGENTINA. Chaco: Herbario de Autran, Feb. 1901, Villa s.n. (6941SI, BAF). Misiones: Dpto. San Ignacio, Teyucuaré, Schinini et al. 21901 (CTES); idem Vanni et al s.n. (CTES). BELIZE. Belize: Cayo rute, P. Gentle 9391 (C); Burrel Boon, Mac Kee 11374 (P); Morazán, near Sta. Clara Vale ni, Yeguare, Standley 23403 (P). BOLIVIA. La Paz: Sud Yungas, NW on Chulumani, S. Rilke 538 (B). Tarija: Arce. Campamento río Negro, T. Meyer 17531 (CTES); De Chiquitos, Weddell 3475 (P). BRAZIL. Commerson s.n. (P); Martius 11215 (P). Bahia: Bahia, en sabulosis marismis, may-II-1900, Salzman s.n. (M); Salzman, s.n. (P); Moricand s.n. (P); Jacobina, M. Blanchet 3874 (P); Ituacu, estrada Ituacu-Barra da Estiva, a $13 \mathrm{~km}$ de Ituacu, próx. do rio Lajero, Giulietti et al. (SPF 18078-CTES); 
R.O. Vanni -The genus Stylosanthes in South America

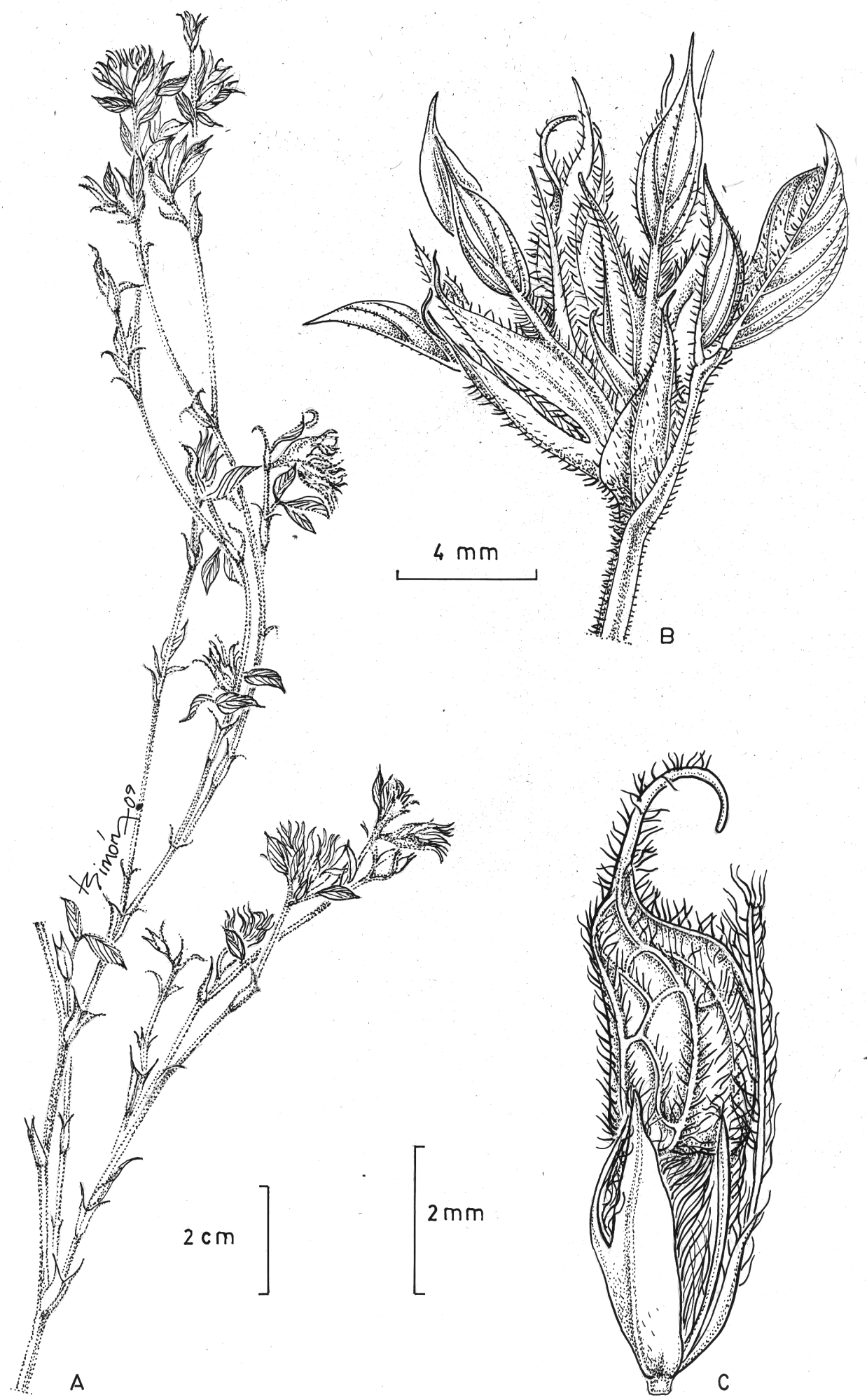

Fig. 12. Stylosanthes tuberculata. A. Plant. B. Inflorescence. C. Fruit. (Liesner 4820). 
18 km SE de Feira de Santana, Krapovickas 12797 (CTES); Lencois, BR 242, 3-8 km W del desvío a Lencois, Arbo et al. 5796 (CTES); 4 km along estrada de terra from Livramento do Brumado to rio de Contas, caatinga, G. Lewis et al. 1912 (NY). Goiás: Formosa, $12 \mathrm{~km} \mathrm{~S}$ de JK, BR 020, 15²'12'S, $15^{\circ} 10^{\prime} \mathrm{W}$, Krapovickas et al. 38685 (CTES). Mato Grosso do Sul: Alto Caracol, Hatschbach 58848 (CTES); Corumbá, $29.5 \mathrm{~km} \mathrm{~S}$ do posto Esdras, estrada do Tamarineiro, A.Pott et al. 7784 (CTES). Minas Gerais: Lagoa Santa, Warming 3177 (C); Serra do Cipo, 10-20 km de Cardeal Mota $19^{\circ} 20^{\prime} \mathrm{S}, 43^{\circ} 35^{\prime} \mathrm{W}$, Arbo et al. 4142 (CTES). Pará: Wullfchlogel 1851 (BR); Paraiba, Areia, J. Vasconcelos 188 (B). Paraná: Paranaguá, T. M. Pedersen 7778 (C); Curitiba, Dombrowski et al. 1126 (CTES). Pernambuco: Prov. Pernambucana, et Bahiensis, Martius s.n. (M); Gravatá, km 96, Estrada BR 232, Krapovickas et al. 38023 (CTES), Distr. Federal, Cabeca do Veado $15^{\circ} 54^{\prime} \mathrm{S}, 47^{\circ} 49^{\prime} \mathrm{W}$, $2 \mathrm{~km}$ sul da Escola Fazendária, Kirkcbride 1626 (CTES). Rio de Janeiro: Warming 3167 (C); Blanchet 31 (P); St. Hilaire cat. A2 N14b $2^{\circ}$ part (P); Godichaud 899 (P); Glaziou 1374(BR); Lagoa Santa, Warming 3179 (C); R.J.F, populis ad Sebastianopolis, Sep. Martius s.n. (M); Recreio dos Bandeirantes, Krapovickas et al. 23203 (CTES); Restinga de Copacabana, Glaziou 5817 (C); Paratí, Krapovickas et al. 35511 (CTES); Guanabara, barra da Tijuca, Z.A. Thiita $524(\mathrm{~K})$. Rio Grande do Sul: Barra do Ribeiro, B. Rambo 46654 (CTES); Brasil. Otto s.n., cited by Taubert. Santa Catarina: Ilha Santa Catarina, Gaudichoud 209(P); Florianópolis, A. Rohr 25384 (B); Acceso a Laguna, Krapovickas et al. 37747 (CTES); Itapema, 14 Jan. 1993, Poletti s.n (CTES). Sao Paulo: St. Hilaire 1669 (P); Regnell 427 (K); Rio Claro, A. Lofgren 575 (C); Itararé, Krapovickas et al. 40928 (CTES); Mun. De Ubatuba, Ubatuba city, Eiten et al. 3334(K). COLOMBIA. Bogotá: Jazagasuga, Hartweg 958 (P). Huila: Cordillera oriental, E of Neiva, Rusby et al. 1087 (NY). Tolima: Tolima, O. Hanght 2444(K), Honda, Pennel 3608(NY). COSTA RICA. Guanacaste: P. Nac. Guanacaste, cerro El Hacha, $11^{\circ} 00^{\prime} \mathrm{N}$, 8332'50"W, A. Chacón 669 (K). CUBA. Ramón de la Sega s.n. (P); Pabana prop. Cayama, Baker 2503 (B); Camgüey Puente de Palo, cerca de Pontezuela, W. Grenter et al. 25127 (B). EL SALVADOR. Usulutan: laguna de Alegria, cerca de los fumaroles, D. Williams 41 (K).GUIANA. Herb. Schomburg 178 (BR); Along the Berbice Rupununi cattle trail, Berbice Demerara country, Abraham 89 (NY); Cayene, Alexandre 417-b (NY); Anglaise, Schomburk 33 (P); Savanna de Crossony, sur la piste de St. Elie, 4-7 km al WSW de Simamary, A. Reynal Roques 20094 (GENT). JAMAICA. Wiellschlaegel 801 (M). MEXICO. Morelos: Yautepec, El Laurel, estación de Microondas, a $13 \mathrm{~km}$ al E-SE de El Camarón, Sousa et al. 9449 (CTES). Veracruz: Manay, Cerro Azul, Mac Kee 10952 (P). PARAGUAY. Amambay: Sierra de Amambay, T. Rojas 10719 (P). Central: In regione lacus Ypacaray, Hassler 11576 (C); Tarumendy, Schinini 6222 (CTES). Concepción: Cursus fluminis E. Hassler 8057 (P); Zwischen, rios Apa Aquidaban, K. Fiebrig 4348 (M). Cordillera: Valenzuela, Mbocaya-guazú, Sparre y Vervoorst 1071 (CTES); Emboscada, Bordas et al. 4256 (CTES). Paraguarí: Paraguarí, sur les collines incultis, B. Balansa $1503^{a}(\mathrm{BR})$; Guazú-rocai, Piribebuy, Schinini 3628 (CTES); Prope Paraguay, Esperanza, Hassler 10719 (NY); In regione Cordillera Centralis, Hassler 6351 (NY). PERU. Cajamarca: Distr. Ichocán, Chancay, Valle Condebamba, I. Sanchez Vega et al. 2427 (BR). SURINAM. Forest of Zandery, Samuels 260 (NY); Afobaka, C.J. Gosteling 45 (GENT); Zanderij I, J. \& P.A. Florschiitz 818 (C). U.S.A. Texas: Aransas, near Aransas County airport, Correl et al. 18899 (CTES). VENEZUELA. Bolivar: Ordaz, Cerro Bolivar, railroad Basset Maguire et al. 36014 (NY); Distr. Piar, hato "Terecay" 16 km N de "El Manteco", O. Huber et al. 6484 (K); Cd. Bolivar hacia Caicara del Orinoco, $7^{\circ} 23^{\prime} \mathrm{N}, 62^{\circ} 27^{\prime} \mathrm{W}$, A. Gröger 1185 (M); Sucre. Península Manare, Steyermark et al. 108030 (NY); Loma Moroton, near Valera, H. Pittier 10721(P).

Obs. The specimen Autran s.n. from Chaco, of which are left only the rest of a plant and a fruit typical of $S$. viscosa, was identified by Burkart (1939) as $S$. viscosa var. acutifolia, but it is in fact a fruit of $S$. viscosa var. viscosa. Although the fruit of $S$. debilis possesses two segments, a feature that can be found also in $S$. viscosa, it displays all the features of the latter species, including the typically elongated inflorescences. Furthermore, the distribution of $S$. debilis as restricted to one state of Brazil is unusual. 


\section{Excluded name}

S. guianensis var. pauciflora Brandao, N. M. Costa \& R. Schultze-Kraft, in Prog. Resum. Soc. Bot. Brazil, XXXVI Congr. Nac. Bot. Curitiba: 323. 1985 , is a nom. nudum.

\section{ACKNOWLEDgeMENTS}

I deeply thank the curators of the herbaria that provided material for this study. This research was kindly supported by the Rupert Barneby Award (1994) of the New York Botanical Garden and by a fellowship of the Myndel Foundation (2008).

\section{BibliogRAPHY}

BLAKE, S.F. 1920. Nine new plants of the genus Stylosanthes. Proc. Biol. Soc. Washington 33: 45-54.

BURKART, A. 1939. Estudios sistemáticos sobre las Leguminosas Hedisareas de la Republica Argentina y regiones adyacentes. Darwiniana 3: 232-261.

BURKART, A. 1967. Leguminosae. En A. L. Cabrera (ed.). Fl. Prov. Buenos Aires, Colecc.Ci. Inst. Nac. Tecnol. Agropecu. 4 (3a): 394-647

CALLES, T. \& R. SCHULTZE-KRAFT. 2010. Reestablishment of Stylosanthes gracilis (Leguminosae) at species level. Kew Bull. 65: 233-240.

FERNÁNDEZ, A. 1973. El acido láctico como fijador cromosómico. Bol. Soc. Argent. Bot. 15: 287-290.

FERREIRA, M. B. \& N. M. SOUSA COSTA. 1977 [1978]. Novas espécies do género Stylosanthes para Minas Gerais. Anais. Soc. Bot. Brasil 28: 81-100.

HASSLER, E. 1919. Novitates paraguarienses, XXIII (cont.) Repert. Spec. Nov. Regni Veg. 16: 220-233.

JANSEN, P. I. \& L. A. EDYE. 1996. Variation within Stylosanthes sp. aff. S.scabra and comparison with its closest allies, S. scabra and S. hamata. Aust. J. Agric. Res. 47: 985-996.
KRAPOVICKAS, A. \& W. C. GREGORY.1994. Taxonomía del género Arachis (Leguminosae). Bonplandia 8: 1-186.

LEWIS, G., B. SCHRIRE, B. MACKINDER \& M LOCK (eds.). 2005. Legumes of the World. Royal Botanic Gardens, Kew.

MAASS, B. L. \& L. 'T MANNETJE. 2002. Stylosanthes seabrana (Leguminosae: Papilionoideae), a New Species from Bahia, Brazil. Novon 12: 497-500.

MOHLENBROCK, R. 1957. A revision of the genus Stylosanthes. Ann. Missouri Bot. Gard. 44: 299-355.

SANTOS-GARCIA, M. O., KARIA, C. T., RESENDE, R. M. S., CHIARI, L., VIEIRA, M. L. C., ZUCCHI, M. I. \& SOUZA, A. P. 2012. Identification of Stylosanthes guianensis varieties using molecular genetic analysis. AoB PLANTS 2012. pls001. doi: 10.1093/aobpla/pls001.

SIMON, M. F., R. GRETHER, L. P. DE QUEIROZ, C. R. SKEMA, T. PENNINGTON \& C. E. HUGHES.2009. Recent assembly of the Cerrado, a neotropical plant diversity hots pot, by in situ evolution of adaptations to fire. Proc. Natl. Acad. Sci. 106 (48): 20359-20364.

SOUSA COSTA, N. \& C. VAN DER BERG. 2003. A new species of Stylosanthes (Leguminosae Papilionoideae), from Mato Grosso do Sul. Kew Bull. 58: 743-747.

TAUBERT, P. 1890. Monographie der Gattung Stylosanthes. Verh. Bot. Vereins Prov. Brandenburg 32: 1-33.

'T MANNETJE, L. 1977. A revision of varieties of Stylosanthes guianensis (Aubl.) Sw. Aust. J. Bot. 25: 347-362.

VANNI, R. O. \& A. FERNANDEZ. 2011. The true identity of Stylosanthes seabrana B. L. Maass \& L. 't Mannetje (Leguminosae Papilionoideae). Caryologia 64: 247-250.

VOGEL, T. 1838. Stylosanthes. Linnaea 12: 63-71.

Recibido el 26 de agosto de 2016, aceptado el 16 de diciembre de 2016. 
